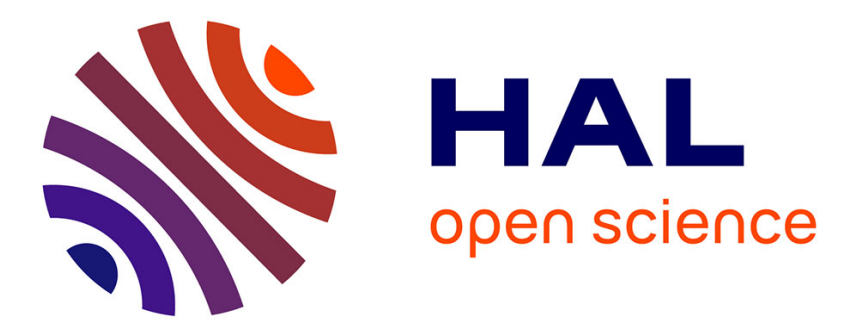

\title{
Three-Dimensional Failure Patterns Around an Inflating Magmatic Chamber
}

\author{
Muriel Gerbault, Riad Hassani, Camila Novoa Lizama, Alban Souche
}

\section{To cite this version:}

Muriel Gerbault, Riad Hassani, Camila Novoa Lizama, Alban Souche. Three-Dimensional Failure Patterns Around an Inflating Magmatic Chamber. Geochemistry, Geophysics, Geosystems, In press, 10.1002/2017GC007174 . hal-01745103

\section{HAL Id: hal-01745103 \\ https://hal.science/hal-01745103}

Submitted on 27 Mar 2018

HAL is a multi-disciplinary open access archive for the deposit and dissemination of scientific research documents, whether they are published or not. The documents may come from teaching and research institutions in France or abroad, or from public or private research centers.
L'archive ouverte pluridisciplinaire HAL, est destinée au dépôt et à la diffusion de documents scientifiques de niveau recherche, publiés ou non, émanant des établissements d'enseignement et de recherche français ou étrangers, des laboratoires publics ou privés. 


\title{
Three dimensional failure patterns around an inflating magmatic chamber
}

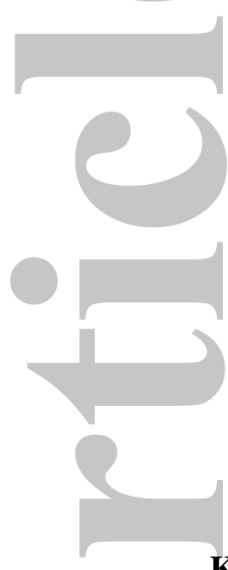

\author{
Muriel Gerbault $^{1}$ (D), Riad Hassani ${ }^{2}$, Camila Novoa Lizama $^{1}$, Alban Souche $^{3}$ \\ ${ }^{1}$ GET IRD UR154, OMP 14 Av. E. Belin, 31400 Toulouse, France \\ ${ }^{2}$ Geoazur, 250 rue A. Einstein, Sophia-Antipolis, France \\ ${ }^{3}$ PGP, Department of Geosciences, University of Oslo, Norway
}

\section{Key Points:}

- The overpressure required for failure at the walls of a magma chamber depends on its geometry and on the effective bedrock strength.

- Plastic shear bands (faults) initiate either from near the apex or the tip depending

on the symmetry and elongation of an oblate chamber.

- Elastic dilation at oblate chambers tip open concurrent magma pathways whereas contraction around prolate chambers favors apex failure.

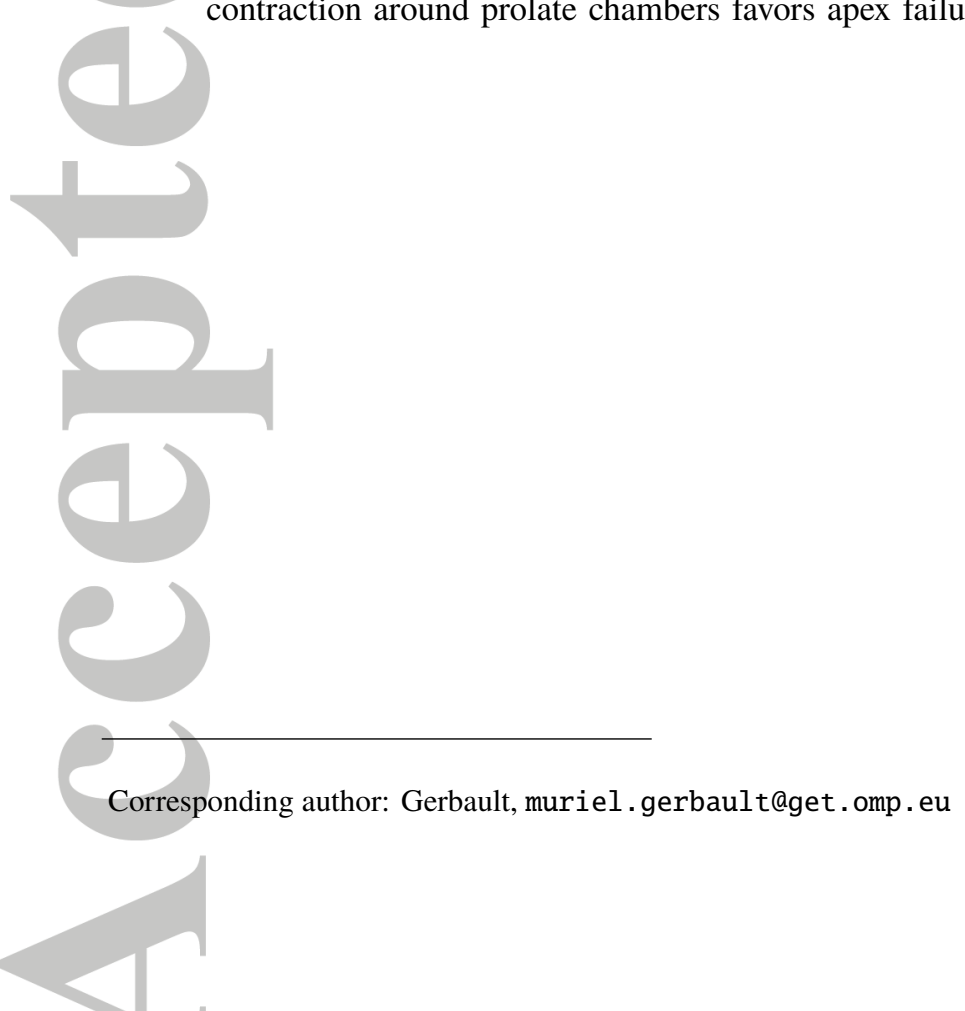

This article has been accepted for publication and undergone full peer review but has not been through the copyediting, typesetting, pagination and proofreading process which may lead to differences between this version and the Version of Record. Please cite this article as doi: 10.1002/2017G C007174

(C) 2018 American Geophysical Union

Received: Aug 02, 2017; Revised: Feb 12, 2018; Accepted: Feb 14, 2018

This article is protected by copyright. All rights reserved. 


\section{Abstract}

Bedrock failure around an inflating magma chamber is an important factor that controls the occurrence of volcanic eruptions. Here, we employ 3D numerical models of elastoplastic shear failure around an inflating crustal reservoir, to study how the induced failure patterns depend on the geometry of the chamber, on the host rock strength and on the gravitational field. Our simulations show that either localized of diffuse plastic failure domains develop in 3 stages. Failure initiates (stage 1) after a critical overpressure is reached, the value of which depends on effective host rock strength. Next, and with increasing applied overpressure, either distributed (for zero friction angle) or localized plastic failure zones (for $30^{\circ}$ friction angle) form (stage 2), until they finally connect to the surface (stage 3). Cylindrical chambers develop prismatic shear zones that simultaneously merge from the surface and chamber walls. For spherical and prolate chambers, diffuse conical zones of failure develop from the chamber's crest, whereas for oblate symmetrical chambers, shear bands initiate at the horizontal tips but bend back above the center of the chamber to reach the surface. In contrast for asymmetrical oblate chambers, shear bands initiate in their cylindrical section and vanish along the elongated direction. Here, magmatic fluids may migrate both through diffuse elastic dilation zones at the tips, and through localized shear zones from the crest. Our results thus suggest that natural observations may be used to constrain the mode of failure occurring underneath a volcano. We discuss several natural examples in this context.

\section{Introduction}

The conditions for which a magmatic chamber can produce a volcanic eruption, and more specifically how magma breaks its way through crustal rocks, remain incompletely understood. This process depends on a number of coupled physical processes at multiple scales in space and time (Karlstrom et al. [2010], Degruyter and Huber [2014], Bonali et al. [2015]). Inside the reservoir, the transfer of magmatic material and heat from deep sources, buoyancy, vapor exsolution and crystallisation all contribute to its internal pressurization. Modelling studies that explore these processes use petrological, structural and chemical data. Outside the reservoir, the response of the surrounding bedrock to variations in internal pressure controls the ability of the reservoir to expand, either as an expanding mush or in a more localised mode along dikes and shear zones that serve as magmatic pathways towards the surface [Anderson, 1936; Petford et al., 2000]. Both mode I 
and mode II magma conduits are observed in the field in a variety of contexts Woodcock and Underhill [1987]; White et al. [2011]; Plateaux et al. [2012]; Spacapan et al [2017]. Several laboratory experiments show that dykes can propagate from self-induced shear failures rather than tensional hydraulic fracturing in the host rock Mathieu et al. [2008]; Abdelmalak et al. [2012]; Haug et al. [2017]. Another type of mechanical approach uses measurements of surface deformation in order to retrieve the geometry and location of magmatic sources at depth, formulating the problem in terms of an inflating or deflating magmatic cavity embedded in an elastic medium [Anderson, 1936; Mogi , 1958; McTigue, 1987; Gudmundsson , 1988; Masterlark, 2007; Karaoglu et al. , 2016; Le Mevel et al. , 2016]. Our approach here is based on this latter view-point, according to which the terms 'reservoir' or magma 'chamber' describe a magmatic fluid source of sufficiently low viscosity so that no significant tangential stresses are applied against its walls. Here, magmatic overpressure is conceptualised as an increasing uniform pressure inside a reservoir of predefined geometry. This overpressure is assumed to increase faster than thermal heat transfer and viscous relaxation, and the reader is refered to other studies that explore such thermal aspects [Gelman et al., 2013; Jellinek and de Paolo , 2003; Burov et al., 2003; Karlstrom et al. , 2010]. The influence of a volcanic edifice, bedrock mechanical heterogeneities and tectonics stresses [Pinel and Jaupart, 2005; Menand, 2011; Currenti and Williams, 2014; Karaoglu et al. , 2016] are also neglected here but will be briefly discussed later on.

The present study aims at assessing 1) the pressure threshold for and 2) the pattern of elasto-plastic deformation in a three-dimensional (3D) bedrock space, extending the two-dimensional (2D) study of Gerbault [2012a]. Here the term 'plasticity' is used in the sense of time-independent plasticity (no viscous effects) to describe brittle failure alone.

The community appears divided on whether it is appropriate or not to account for the gravity body force in numerical models of volcanic inflation (Grosfils et al. [2015]). Obviously, gravity plays a first order role on bedrock failure conditions [Gerbault, 2012a], on the geometry of magma propagation with respect to an edifice load [Corbi et al., 2015], and on buoyancy contrasts driving magma upward [Lister and Kerr, 1991; Watanabe et al. , 2002]. However, many models neglect the gravitational loading of the bedrock itself, assuming that volcanic deformation and eruptions result from a change with respect to a stage previously at equilibrium and without deformation; therefore the influence of the isotropic component of the stress field is supposed to cancel out. Actually neglecting grav- 
itational loading is implicitely equivalent to the assumption that the crust has lithostatic fluid pore-pressure everywhere. The validity and implications of this assumption have been recently thoroughly discussed in Grosfils et al. [2015], and it is not the aim of the present study to debate this. Nevertheless, in the aim of bridging the gap between communities we shall present here models with and without gravity, and with and without friction. A situation in which gravity is neglected thus aproximates a bedrock with lithostatic pore-fluid pressure everywhere, whereas a case with gravity and zero friction angle approximates a dry crust that has been thoroughly weakened by multiple fractures and mobilization [Ikari et al., 2009], End-member models without friction and without gravity constitute extreme cases where the bedrock fails at a minimum applied internal pressure. On the other hand, cases with friction and gravity correspond to standard dry resistant bedrock, that fails at maximal internal pressure conditions.

The questions adressed here are: 1) can 3D failure patterns be directly deduced from 2D predictions? and 2) what can such predictions of 3D deformation patterns and pressure conditions teach us about the underlying magma reservoir geometry? In the following, we first recall previous predictions of the critical overpressure leading to bedrock failure around cylindrical (2D) and spherical (3D) magma chambers. Next we present our numerical method and reproduce in 3D previously published 2D results. We display a comparison of the overpressure necessary for the onset of shear failure at the chambers walls for various oblate to prolate geometries, using the study of Currenti and Williams [2014] and an alternative code (A. Souche, pers.comm.). Section 3 details the evolution of the stress and strain fields with increasing overpressure, for spherical, oblate and prolate reservoirs. Cases without friction and without gravity are also discussed. We finally comment on the influence of these chambers geometries on surface deformation, on the conditions for mode I versus mode II failure, and on how magmatic pathways may be influenced by such patterns of stress and deformation. We briefly review other parameters that may play a role as well and draw some implications for natural volcanic systems.

\section{Previous predictions of failure initiation, mechanical model and numerical method}

\subsection{Analytical predictions for bedrock failure}

The stress and strain fields resulting from an inflating spherical source have been studied for nearly a century, since pioneer works in an elastic half space (Jeffery [1921]; 
Anderson [1936]; Jeffery [1921]; Mogi [1958]). It is (still) commonly assumed that tensile failure (mode I) initiates along the walls of a magmatic chamber submitted to an internal overpressure (radial $\Delta P$ ), in locations where the tangential stress is minimal (or hoop stress $\sigma_{\theta \theta}$, defined as the normal stress perpendicular to the radial direction acting in the tangential plane, Jeffery [1921]; McTigue [1987]). Here, overpressure $\Delta P$ is the pressure in excess of the lithostatic pressure $P_{l}=\rho g z$ (at depth $z$, negative downward). For a spherical chamber, the critical overpressure is expressed as a function of the chambers depth $H$ and radius $R$, and the bedrock's tensile strength $T_{o}$ (e.g. Pinel and Jaupart [2005]):

$$
\Delta P_{T}=\frac{H^{2}-R^{2}}{H^{2}} \cdot T_{o}
$$

Grosfils [2007] demonstrated the necessity of accounting for gravity when assessing the conditions for tensile failure, since indeed the force balance involves a gravitydependent hoop stress that does not cancel out. Consequently when accounting for a gravitationally loaded half space, much larger critical overpressure is required for the onset of failure at the chamber walls. Grosfils [2007] predicted that this critical overpressure depends on depth $z$ and on the free surface shape factor $C_{1}=1+2 \tan ^{2} \alpha$ ( $\alpha$ the angle measured from the origin at the surface and between the vertical axis and a point along the chamber, Jeffery [1921]) :

$$
\Delta P_{T G}=\frac{2}{C_{1}} \cdot\left(T_{o}-\rho g z\right) .
$$

For ellipsoidal magma chambers embedded in a gravity field, Grosfils [2007] predicted that vertical dikes tend to form at the apex of spherical and prolate chambers at depths greater than $2 \mathrm{~km}$, whereas lateral sills form instead around oblate chambers deeper than 1 $\mathrm{km}$.

With gravity and in 2D, Gerbault [2012a] investigated the conditions for either tensile (mode I, hydrofracture) or shear failure (mode II, Coulomb type), by modeling the evolution of deformation and failure around a progressively increasing internal pressure. Gerbault et al. [2012] fitted tensile failure predictions by Grosfils [2007], however shear failure was actually found to be triggered prior to tensile failure at the chamber walls, unless the bedrock has a lithostatic pore fluid pressure. In that case, the liquid pressure inside the pores of the bedrock exerts a resisting buoyancy force to the ambient normal component of the stress tensor [Hubbert and Rubey, 1959], which thus cancels its influence on the yield threshold. Even a medium at an intermediate state of hydrostatic pore 
fluid pressure was found to still fail under mode II prior to mode I at the walls of the chamber. Gerbault [2012a] evaluated (in 2D), the critical overpressure $\Delta P_{M C S}$ necessary to reach the Coulomb yield envelope at any point at depth $z$ along the chamber wall, as a function of bedrock friction $\phi_{o}$, cohesion $C_{o}$ or tensile strength $T_{o}\left(C_{o}=T_{o} \cdot \tan \phi_{o}\right)$, and the free surface factor $C_{1}$ :

$$
\Delta P_{M C S}=2 \frac{\sin \phi_{o}}{1+\sin \phi_{o}+C_{1} \cdot\left(1-\sin \phi_{o}\right)} \cdot\left(T_{o}-\rho g z\right) .
$$

When failure occurs at the apex, $\alpha=0, C_{1}=1$ and $\Delta P_{M C S}=\left(T_{o}-\rho g z\right) \cdot \sin \phi_{o}$.

In an elastic 3D medium, the stress balance at a spherical cavity wall requires that the tangential stress is half the exerted outward normal pressure $\left(J_{2}=\Delta P / 2\right.$, [Timoshenko and Goodier, 1970], as opposed to a cylindrical cavity where they become equal $\left(J_{2}=\Delta P\right)$. Consequently, Gerbault [2012a] predicted that for a sphere, the critical overpressure for the initiation of shear failure would be approximately multiplied by 2 compared to plane-strain conditions. Currenti and Williams [2014] obtained slightly lower values. For comparison, the state of stress produced by a spherical inflation in an infinite 3D elastic medium is available from e.g. Timoshenko and Goodier [1970]: the surrounding external pressure is null along the chamber walls, cancelled out by the hoop and shear stresses. If the medium is elasto-plastic with a Tresca yield stress $k$, a spherical plastic zone of radius $c$ develops and increases with increasing internal pressure $\Delta P$. The resulting overpressure $P_{p}$ inside the plastic domain $(r<c)$ relates to the applied $\Delta P$ as:

$$
P_{p}(r<c)=2 \cdot k \cdot\left(\ln \frac{r}{R}+\frac{1}{3}\right)-\Delta P=2 \cdot k \cdot\left(\ln \frac{r}{c}\right) .
$$

We deduce a crude estimate of the state of pressure necessary to generate shear failure (mode II) at the chamber wall $(\Delta P=2 k / 3)$ up to the surface $(\Delta P=2 k(\ln H / R+1 / 3))$. Note that a Tresca yield criterion corresponds to a Coulomb failure criterion with friction $\phi_{o}=0$ and cohesion $C_{o}=k / 2$. We will compare and discuss our numerical results with these values in section 3 below.

\subsection{Mechanical model, numerical setup and method}

The mechanical model, its governing equations and the elasto-plastic constitutive law are provided in Supplementary material. Below we describe the initial geometry of the model domain and our choice of boundary conditions. Our numerical method, Adeli, is also presented. 


\section{Initial geometry and boundary conditions}

Because of obvious symmetry, the model's initial geometry covers only a quarter of domain $\Omega$. It is defined as a cubic domain of side length $L$, containing an ellipsoidal cavity of principal axes parallel to the coordinates axes and centered at point $(0,0,-H)$ (Fig. 1). The vertical semi-axis ( $z$ direction) of this cavity is denoted by $a$, the two horizontal semi-axes ( $x$ and $y$ directions, respectively) by $b$ and $c$. Boundary conditions are:

- the top ground surface (initially at $z=0$ ) is a stress-free surface: $\sigma \cdot \mathbf{n}=\mathbf{0}$,

- the vertical boundaries (at $x=0, x=L, y=0$ and $y=L$ ) and the bottom boundary $(z=-L)$ are free-slip and held stationnary in their normal direction: $\mathbf{v}^{t} \mathbf{n}=0$ and $\sigma_{t}=0$ (where $\sigma_{t}=(I-\mathbf{n} \otimes \mathbf{n}) \sigma \cdot \mathbf{n}$ is the tangential stress vector),

- the curved wall chamber boundary is submitted to a normal stress corresponding to the sum of lithostatic pressure $p_{l}=\rho g|z|$ (where $g=\|\mathbf{g}\|$ ) and an overpressure $\Delta P$ : $\sigma \cdot \mathbf{n}=-\left(p_{l}+\Delta P\right) \mathbf{n}$.

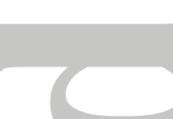

All models are initialised with isotropic lithostatic stress components everywhere (the weight of overburden rock $\rho g z$ ), a uniform density $\rho=2500 \mathrm{~kg} \mathrm{~m}^{-3}$, and gravity, set either to $g=0$ or $g=10 \mathrm{~m} / \mathrm{s}^{2}$. Thus models begin at $\Delta P=0$ at equilibrium, e.g. with no initial deformation. Throughout this study, tensile stresses are defined positive.

In the models, the abrupt application of a normal load on the chamber walls can locally exceed the brittle yield stress and thus lead to unrealistic distorsions. Therefore the internal overpressure $\Delta P$ is applied in incremental steps $\delta p$, large enough for the total computing time to remain reasonable, but small enough to achieve non-linear convergence during each pressure increment (as in Gerbault et al. [2012]). Consequently our model results below have an uncertainty in $\Delta P$ of about $\simeq 10 \%$ corresponding to the step-wise pressure increment and to the sampling frequency of the output.

The mesh is defined with tetrahedral elements of length size $25 \mathrm{~m}$ in the first 10 $\mathrm{km}^{3}$ of the domain, departing at coordinate $(0,0,0)$. Grid resolution progressively reduces in all directions to a maximum size of $10 \mathrm{~km}$ at the opposite corner of the mesh-domain located at coordinate $(80,80,-80) \mathrm{km}$. The total number of elements is on the order of $2-6 \times 10^{6}$ depending on magma chambers geometries.

A reference spherical chamber model is set up with a center depth $H=7 \mathrm{~km}$ and radius $R=2 \mathrm{~km}$. Relative to this case, the shape of oblate chambers is explored while 
maintaining a constant vertical axis $a=R$ and varying the horizontal axes $b$ and $c$. Sym-

metrical oblate chambers have two elongated horizontal axes (pancake shape), while asymmetrical oblate chambers have only one elongated axis $c$, along direction $y$ (in the two other directions $b=a=R=2 \mathrm{~km}$ ). Prolate chambers in turn are studied with constant horizontal axes $(b=c=R)$, and a variable vertical $a$ axis (cigar shape).

\section{Numerical method}

An approximate solution to the continuous problem (Eq. S1-1) is computed using the finite element code Adeli [Hassani et al., 1997]. Adeli has been used to simulate a variety of geodynamical contexts, including subduction zones [Hassani et al., 1997; Cerpa et al., 2015] and the seismic cycle [He and Chéry, 2008; Contreras et al., 2016]. This code uses the dynamic relaxation method (Cundall and Board [1988], Cundall [1989]) to solve the set of non linear equations obtained after a finite element discretization of the quasi-static equilibrium equation. This time-explicit method uses an adaptative time-step and basically damps the out-of-balance forces; it was designed specifically to track the development of localised plastic strain. It has been widely tested in the engineering and academic Earth Sciences communities, as a product of the commercial Itasca Consulting Group (FLAC code), and via academic codes such as Parovoz [Poliakov et al., 1993; Gerbault et al., 1998; Burov et al., 2003] (or Flammar later on) and Adeli. In Adeli, common time-integration algorithms like elastic prediction-plastic correction [Ortiz and Simo, 1986] are used to compute the current stress state. More details about numerical aspects can be found in Hassani and Chéry [1996]; Chéry et al. [2001]; Cerpa et al. [2015].

\subsection{D behavior}

In plane-strain, with gravity and $\phi=30^{\circ}$, three stages characterise the development of plastic deformation with increasing overpressure (Fig. 2c, Gerbault et al. [2012]), and with which we subsequently compare our 3D models :

- Stage 1 , failure initiates at the surface above the center of the magmatic chamber,

- Stage 2 shear failure initiates along the chamber wall (at overpressure satisfying Eq.3. Shear zones develop oriented $\sim 60^{\circ}$ apart, and depart at about a quarter arc distance from the chamber's apex, consistent with predictions (Grosfils [2007]; Gerbault [2012a]). They propagate away and upward with a radial-excentric geom- 
etry (a term that describes the slip lines obtained by Nadai [1931]; d'Escatha and Mandel [1974]). Both the upper plastic domain at the surface, and the excentric plastic domain at the chamber walls, form two conical domains that progressively expand towards each-other.

- Stage 3 is defined with the connection of these two conical plastic domains. Shear bands continue to expand outwards from the chamber, and an increasing number of shear zones develop, directly connecting the wall's lateral edges to the surface. At this stage the mechanical connection between the chamber and surface is obvious. This geometry is consistent with solutions obtained in tunneling mechanics (Gerbault et al. [2012], and references therein). This stage 3 is likely beyond the eruptive stage of a natural volcanic system, yet it constitutes an upper limit for its general failure.

Figures 2 illustrate four models of two-dimensional reservoirs in three dimensions (equivalent to plane-strain conditions), testing the influence of $\phi$ and gravity. For each of these cases the figures display three stages of evolution of the plastic domain $\left(\varepsilon_{p}\right)$ :

- The first model case has a friction angle $\phi=0$ and gravity (equivalent to a von Mises criterion Fig. 2.a). Plastic failure develops first at the wall at $\Delta P_{1}=12 \mathrm{MPa}$, consistent with Eq. 1, and then at the surface. Connection occurs after $\Delta P_{3}=29.5$ MPa (stage 3).

- The second case has friction $\phi=30^{\circ}$ and no gravity $(g=0)$, equivalent to a Coulomb-type bedrock at a state of lithostatic state pore-fluid pressure (Figure 2.b). The evolutionary stages are identical, but excentric shear bands develop at depth and a plastic cone forms at the surface bounded by a sharp $30^{\circ}$ to $45^{\circ}$ dipping shear band. Critical overpressures are obtained of the same order $\left(\Delta P_{1}=12\right.$ $\left.\mathrm{MPa}, \Delta P_{3}=32 \mathrm{MPa}\right)$.

- The third case with friction $\phi=30^{\circ}$ and gravity accounted for, refers to the standard situation of dry bedrock. Failure initiates at the surface (stage 1), then at the apex of the chamber wall (stage 2), and these two plastic domains connect (stage 3), similarly to Gerbault et al. [2012]. Critical overpressures now exceed $100 \mathrm{MPa}$ (Table 1).

- The last case displays an oblate-cylindrical chamber with $b=7 \mathrm{~km}$, gravity and $\phi=30^{\circ}$. Plasticity initiates at the surface, with an expanding conical block. It 
then affects the chamber's tip at $7 \mathrm{~km}$ depth, progressively forming a major inclined shear band that reaches the surface at c.a. $x \simeq 10 \mathrm{~km}$. Critical overpressures reach now $\Delta P_{1}=15 \mathrm{MPa}$ and $\Delta P_{3}=60 \mathrm{MPa}$.

The above first two model cases without gravity reach the yield envelope along the chamber walls first, whereas the two last cases with gravity fail first at the surface, as expected. The three circular-cylindrical cases see their plastic domain connect the chamber and the surface along the $y=0$ plane, forming the typical 2D conical domains on top of radial outward (excentric) domains. Localised structures only appear when $\phi=30^{\circ}$, consistent with non-associated plasticity [Vermeer and de Borst, 1984; Gerbault et al., 1998; Nadai, $1931]$.

The plastic domain evolution around an oblate chamber is noteworthy: while the plastic shear domain initiates from the surface center progressively downward, the final connection of the plastic domain from the chamber to the surface occurs via the tip. This 'outwards-subvertical' geometry of shear failure is consistent with previous predictions of tensile failure (e.g. Anderson [1936]; Gudmundsson [2006]) based on the coinciding location of the maximum intensity of the minimum principal stress $\sigma_{3}$ and the orientation of maximum principal stress $\sigma_{1}$. We shall see that in 3D this situation becomes more complex, and for sake of discussion clarity below, we will further refer to this conventional prediction as the " $\sigma_{3} / \sigma_{1}$ rule".

\section{Three-dimensional geometries and the development of shear failure}

Next we study the evolution of elasto-plastic behaviour in 3D. Below, we start with a comparative study that evaluates the critical overpressure necessary to reach wall shear failure as a function of the chamber's geometrical characteristics. Then for each spherical, oblate and prolate cases we describe the evolution pattern of the stress and deformation fields, the plastic domain and the associated internal overpressure. A final series of tests presents a comparison of surface deformation according to different geometries.

\subsection{Comparative study}

A comparison of the critical overpressure for the onset of shear failure is performed against the study of Currenti and Williams [2014], in order to check the robustness of the results obtained with Adeli. In addition, we present numerical calculations obtained with 
another implicit finite element method (developped by A. Souche, pers.comm., www.bitbucket.org)

that allows for a finer spatial discretization of the chamber walls. For this purpose, the model parameters are set accordingly to those used by Currenti and Williams [2014], which we refer to as the "CW models": Young's modulus $E=75 \mathrm{GPa}$, density $\rho=2600 \mathrm{~kg} / \mathrm{m}^{3}$, $\phi=35^{\circ}, C=5 \mathrm{MPa}$. We also report the results obtained with $R=2 \mathrm{~km}$ corresponding to our main modeled case, with elasto-plastic properties described in the setup section.

The critical overpressures obtained with Adeli and for different depths $H$, are reported in Table 1 and displayed Fig. 3a. Our results fall in between those of Currenti and Williams [2014] and the approximation by Gerbault [2012a] (Eq.3 multiplied by 2, shift from $2 \mathrm{D}$ to $3 \mathrm{D})$. The critical overpressure $\Delta P$ varies linearly with increasing chamber's depth.

The results obtained by A. Souche in Fig.3a (labelled Adhoc-low res. and Adhochigh res.) were obtained with a mesh discretization at the wall boundary of $70 \mathrm{~m}$ and 8.6 $\mathrm{m}$ respectively, and indicate that a lower model resolution leads to an underestimation of the critical overpressure needed for failure. Numerical accuracy could be gained by using even finer mesh discretization, which we haven't used given the computational cost of 3D calculations. Another comparison was performed with A. Souche's code assuming either a fixed or a mobile wall boundary (meaning either prescribed zero motion or free motion). These two tests did not show noticable variations of the critical overpressure, allowing to conclude that for this mechanical configuration at least, the fixed wall boundary condition remains valid within the early plastic deformation stages (stages 1 to 2). All subsequent results developed with Adeli were obtained by considering a mobile wall boundary.

The comparison of critical overpressures as a function of the chamber's shape ratio $\lambda=b / a$, is displayed for 2 different depths $H=0.5$ and $H=2 \mathrm{~km}$ in Fig. $3 \mathrm{~b}$. The Adeli models slightly overestimate $\Delta P$ with respect to the other approaches for very elongated geometries, but it matches the trend that maximum overpressure is required for nearly spherical chambers $(\lambda \simeq 1.2)$. This suggests that in nature spherical magma reservoirs might not be the most appropriate geometry to trigger an eruption. However this is a commonly used geometry when interpreting measured surface deformation [Mogi , 1958; McTigue, 1987; Masterlark, 2007; Pinel and Jaupart, 2005; Karlstrom et al. , 2010]. 


\subsection{Spherical chambers}

The reference spherical model is displayed Fig. 4a, with gravity and friction $\phi=0^{\circ}$. Failure initiates simultaneously around the chamber walls, at $\Delta P \simeq 13.8 \mathrm{MPa}$ (labelled $\Delta P_{1}$ in Table 1) and fits eq.4 with $r=R=2 \mathrm{~km}$, and $k=20 \mathrm{MPa}$. This plastic domain then expands radially and reaches the surface at $\Delta P=58 \mathrm{MPa}$ (stage 3, labelled $\Delta P_{3}$ in Table 1). This modeled pressure can be considered to match the prediction of $63 \mathrm{MPa}$ provided by Eq.4 for a plastic domain of radius $r=H=7 \mathrm{~km}$, as it stands within the $\simeq 10 \%$ uncertainty of the applied numerical step-wise pressure increment specified above. The slight difference between both solutions may be attributed to the presence of the free surface that impedes the medium to store stresses above the top surface, as opposed to an infinitely wide medium. Note also that until stage 3 , the plastic domain is embedded in the elastic domain so that elastic solutions for surface displacements are still a valid approximation (as shown by Gerbault et al. [1998] and Gerbault [2012a]).

With bedrock friction $\phi=30^{\circ}$ and no gravity (Fig. $4 \mathrm{~b}$, assimilated here to a state of lithostatic pore pressure in the bedrock), the plastic domain around the inflating sphere expands radially as well, and at similar overpressures to the preceding case. However now, both plastic domains merge at about $2 \mathrm{~km}$ depth above the chamber's central axis, as opposed to the case with $\phi=0$ and no gravity, where the plastic domain connects at a distance closer to the surface and at ca. $2 \mathrm{~km}$ away from the central vertical axis.

With bedrock friction $\phi=30^{\circ}$ and gravity (Fig. 4c), the inflating spherical chamber fails first at its apex and then at the surface, in reversed order to a cylindrical chamber. An overpressure $\Delta P \simeq 120 \mathrm{MPa}$ is required for failure to initiate at the chamber walls, whereas only $105 \mathrm{MPa}$ were required in the cylindrical case. This difference is justified by the fact that overpressure $\Delta P$ against the internal walls of a sphere produces less volumetric increase $(\Delta V)$ than against an infinitely long cylinder, explaining the necessity of greater loading to reach the failure threshold at the surface.

During stage 2 when overpressure progressively increases in the spherical chamber, we note that maximum values of $\sigma_{3}$ develop along the lower half domain of the chamber walls and that the orientations of $\sigma_{1}$ point radially outwards (Figs. 5), thus suggesting that diking should develop from these deepest edges according to the " $\sigma_{3} / \sigma_{1}$ rule" mentionned above. However, we also note that the upper half of the sphere develops contraction in a plastic regime (blue domain in mid panel Fig.5b,c), which logically impedes any tensile 
opening there. Consequently this explains why the onset of failure actually occurs near the apex of the chamber.

In the spherical chamber, stage 3 describing the connection of the plastic domain from the chamber to the surface, occurs at an overpressure $\Delta P \simeq 420 \mathrm{MPa}$ (Fig.5c), much greater than for a cylindrical chamber $(\Delta P \simeq 160 \mathrm{MPa}$, Table 1, Fig.5a). Here, both plastic domains expanding from the chamber walls upward and from the surface downward slowly converge, revealing the kinematics related to this mechanical system: shear senses that border the upward moving deepest plastic cone immediately above the chamber (contracting domain), converge and push inward from below, while shear senses that border the shallowest plastic cone diverge upward (inward dipping fault planes, dilating domain).

\subsection{Oblate geometries}

Now the chamber is set elongated horizontally, and we distinguish symmetrical and asymmetrical oblate geometries. Symmetrical chambers have equal horizontal axes $b=c$. Asymmetrical chambers are only elongated along their $c$ axis in the $y$ direction, while their shape remains circular in the $y=0$ plane with axis $b=a=2 \mathrm{~km}$.

In all cases now, shear failure initiates closer to the elongated horizontal extremity of the chamber, confirming the prediction based on the standard $\sigma_{3} / \sigma_{1}$ rule without gravity, and those accounting for gravity [Grosfils , 2007; Currenti and Williams, 2014]. This hints that internal magmatic fluids may tend to expel via these lateral edges of the chamber. The development of failure at these lateral tips is a logical consequence of prevailing tensile conditions induced by the application of a uniform load along sharply curved walls. Hence dilation occurs in the bedrock surrounding these tips (cf. red areas in mid panel Figs. $6 \mathrm{~b}$ and $7 \mathrm{~b}$ ). We shall see below that the subsequent evolution pattern may however, become complicated.

For symmetrical geometries, two cases with $b=c=4,7 \mathrm{~km}$ are displayed (Table 1, Figs. 6b-c), which can be understood as intermediate cases to the spherical and cylindrical oblate end-member cases (Figs. 5b and 6a). As the horizontal axis decreases from 7 to 4 $\mathrm{km}$, the critical overpressure for wall failure increases $\left(\Delta P_{1}\right.$, Table 1$)$, plasticity initiates at the tip for a greater overpressure, and the plastic domain tends to bend back towards the centre above the chamber. The plastic domain at the surface expands radially, and no clear 
shear bands manage to localise since they join at a central point at mid-depth (between 1 and $4 \mathrm{~km}$ depth).

For asymmetrical geometries, three cases with $c=4,7$ and $20 \mathrm{~km}$ (Table 1, Figs.

7) show another type of development of plasticity, also in between the spherical and the cylindrical end-members. First, plasticity develops for smaller overpressure values with increasing chamber's elongation (increasing axis $c$ ). Second, shear bands form at depth along the $y=0$ plane, more localised as the chambers's elongation increases. Shear zones initiating close to the vertical axis produce inward dipping fault planes, and they straighten up as they expand towards the elongated axis (they become more rectilinear in the increasing $y$ direction). It might be useful in specific studies, to track how uplifting and subsiding areas dip and rotate along fault planes with depth, in order to identify the chamber's geometry. Such inclined structures may appear challenging to track from field exposure and to attribute to a simple consistent tectonic stress field, in particular at the depth where compressional and tensile stress fields merge (blue to red transition in mid panels Figs.7).

A key result from the asymmetrical models is that, whereas shear failure initiates at the chamber's tip (elongated axis $c$ in the $x=0$ plane), it remains confined there. Meanwhile the plastic domain develops along the vertical plane $y=0$ where the chamber's wall is circular, connecting the chamber and the surface. In fact, while the tip of the chamber is under a dilating regime, $\sigma_{3}$ is not maximum there, but is maximum instead around the circular section in the vertical plane $y=0$ (Fig. 7 right panels). Note also that, although shear failure occurs at the chamber wall in similar locations to those predicted for tensile failure, (at an inclined angle from the vertical, cf. Grosfils [2007]), it then follows a track oriented at $30^{\circ}$ from $\sigma_{1}$ (in contrast, a dike would be oriented parallel to $\sigma_{1}$ ).

\subsection{Prolate geometries}

Now we discuss cases of prolate chambers with elongated vertical axis $a$ and fixed horizontal axes $b=c=2 \mathrm{~km}$ (Figs. 8, Table 1). A main case has depth $H=9 \mathrm{~km}$ and axis $a=4 \mathrm{~km}$, so that the apex remains at $5 \mathrm{~km}$ depth for comparison with previous geometries. Other cases test depths $H=7,12,23 \mathrm{~km}$ and axis $a=7,20 \mathrm{~km}$, with and without gravity (Table 1).

Whatever the elongation or whether gravity is accounted for or not, the onset of failure always occur at the chamber's apex, in consistency with other predictions [Grosfils, 
2007; Currenti and Williams, 2014]. Without gravity, both the apex and bottom tip fail simultaneously (Fig. 8a). Then, during stage 2 an amphora-shaped plastic domain develops, with a cone of diffuse plastic strain at the surface (dilating domain, Fig. 8b), similar to the spherical case.

When considering the deformation and principal stress field (Fig. 8b,c), we note that the sub-vertical walls of these prolate chambers are under a compressional state of stress, in contrast to tension developing at the horizontal edges of oblate chambers. Such contraction should impede the development of radiating dikes, despite an appropriate location and magnitude of the principal stresses $\left(\sigma_{3} / \sigma_{1}\right.$ rule, discussed further below).

When considering the plastic pattern produced with different prolate geometries, we note that the plastic domain connects (stage 3) at less overpressure for a less elongated chamber, for a shallower apex, and of course, when gravity is not accounted for (Figs. 8, Table 1). Around very elongated chambers, the connection of the plastic domain to the surface requires high overpressure, even without gravity (Table 1 ). Yet we note that $\sigma_{3}$ along the wall is minimum right at the vertical tip (Fig. 8c) right), in apparent contradiction with the classical prediction of tensile failure there.

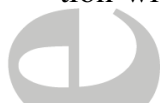

\subsection{Conditions for localised deformation}

Non-associated plasticity, which refers to material properties of differing friction and dilatancy angles, is a necessary condition for deformation to localise in a uniformly loaded, homogeneous medium [Nadai, 1931; Vermeer and de Borst, 1984]. In rocks, the friction angle approaches $30^{\circ}$ while the dilatancy angle approaches $0^{\circ}$. As a confirmation, in all our models thin localised shear bands only form when the friction angle is non-zero. More specifically, localised shear bands form at depth and relate the chamber walls to the surface in cases when the chamber is sufficiently oblate. The plastic domain remains diffuse instead when the chamber's geometry is prolate.

During the intermediate stage 2 above prolate chambers, an annular ring of plastic shear failure develops at the surface at some distance from the chamber's center (Fig. 9a). We find that this zone of plastic shear becomes perceptible at the surface as when approaches the value $H-b$, but this remains to be confirmed by complementary tests covering a full range of geometries. It results from a radial contraction exerted in the horizontal plane by the chamber's overpressure, which propagates upward to the surface. If such an 
annular plastic shear zone (perhaps ring or concentric fault zone) is observed by geodetic measurements above natural magmatic chambers, it may hint to a vertically elongated magma reservoir at depth (perhaps a plug intrusion,e.g. Dieterich and Decker [1975]).

Some of our models also show the development of localised excentric shear bands at the surface, however at overpressures significantly beyond stage 3 . Such overpressures might be considered unrealistic under dry bedrock conditions, since they exceed by a factor of about 5 the standard values derived from estimates for natural volcanic systems, which are generally estimated not to exceed 100 MPa [Masterlark, 2007; Jellinek and de Paolo , 2003; Karlstrom et al. , 2010]. In our model of an inflating sphere with $\phi=30^{\circ}$ and zero gravity, overpressures of about $120 \mathrm{MPa}$ generate excentric shear bands at the surface (Fig. 9c). These structures deserve to be studied in greater detail since they are reported in many places worldwide as well as on other planets (Ernst et al. [1995]; Minakov et al. [2016]).

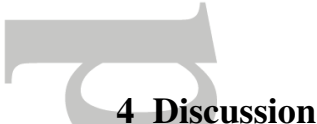

\section{Discussion}

Below, we further discuss three points that our modeling results raise specifically: the conditions for either mode I or mode II failure around oblate chambers (section 4.1), the dependency of surface deformation on 3D chamber geometry, with a discussion example from the Laguna del Maule, Chile (sections 4.2 and 4.3), and the potential influence of our modeled 3D stress and strain fields on magmatic pathways (section 4.4). We discuss these points mentioning briefly specific areas, and we make more general comments on other aspects controling magma propagation from inflating volcanic reservoirs (section 4.5).

\subsection{Tip or top failure around oblate chambers}

Our numerical study above does not account for the occurrence of tensile failure. Since it had been shown that the shear failure envelope is reached prior to the tensile yield in cylindrical, and by extension to spherical geometries (and with gravity, Gerbault [2012a]), we assumed that to a first order, the same situation would occur in ellipsoidal geometries. Yet this remains to be demonstrated specifically at the tip of ellipsoidal chambers. Many approaches based on the theory of elasticity assume that tensile failure occurs in locations where the principal stress $\sigma_{3}$ is maximum and in a direction parallel to $\sigma_{1}$ 
(e.g. Anderson [1936]; Gudmundsson [2006]). When plotting these fields in our models we have seen that (i) shear failure does not always initiate in such domains, and (ii) that the location of maximum $\sigma_{3}$ does not always occur at the tip of 3D oblate chambers (e.g. Fig. 7), thus questioning whether mode I failure at the tip of magma chambers is the main mechanism by which magma migrates and potentially erupts at the surface.

The development of a plastic shear zone from the tip of an oblate chamber, either straight up outwards or instead curving back towards the center up to the surface, also likely depends on the depth/shape ratio $\mathrm{H} / \mathrm{R}$ of the magma chamber. We may gain insight on this from an evaluation by Salençon [1969], based on plastic slip-lines theory: the slip-lines departing from a pressurizing indenter of half-width $\mathrm{R}$ can intersect prior to reaching the free-surface at a maximum distance $H / R>5.29$. We thus guess that oblate magma chambers deep enough will rather produce intersecting slip-lines above their apex instead of failing at their lateral edges. We may extend this reasoning and propose that, conversely, there should be a critical width-to-depth ratio of a magma chamber, beyond which outwards sub-vertical shear zones will connect the lateral tips to the surface. Such a magic number would also depend on the third axis of the magma chamber, thus requiring another set of tests which we choose not to explore in this already lengthy contribution. As an example, the Torres del Paine pluton, Chile, is a well preserved oblate crustal magma body that has been exposed by glacial erosion. Many dikes protrude from the chamber walls into the host rock, with in appearance a higher concentration at the tip than at the roof of the pluton [Skarmeta and Castelli, 1997; Leuthold et al. , 2012]. However, a large portion of the roof is actually missing. If this pluton can be assimilated to an inflating oblate magma chamber, we may then propose to interpret the dikes at its tip as aborted structures, and that magmatic fluids in the reservoir either further migrated via failure pathways developping from the roof (however vertical magmatic flow is not evident from field data), or that they stagnated within the reservoir, progressively cooling down without further expansion. This behaviour is consistent with the rather asymmetrical oblate shape of the Torres del Paine pluton (horizontal axes of about 7 and $17 \mathrm{~km}$ ).

\subsection{Surface deformation and 3D chamber geometry}

Forty years ago, Dieterich and Decker [1975] numerically modeled the elastic deformation produced by inflating spherical and non spherical chambers, concluding that differ- 
ent patterns of surface displacements allow to identify different source geometries. Today, this issue remains crucial as pointed by Remy et al. [2014], who showed that a sphere, a prolate ellipsoid or a planar crack located at different depths can all explain the surface displacements measured at Lazufre Volcano, Chile. In order to help decipher between possible reservoir geometries, we present two series of comparisons below.

A first series of tests displays surface displacements for a given arbitrary overpressure $\Delta P=150 \mathrm{MPa}$. Fig. 10a shows the resulting surface displacements and plastic strain for a sphere, a cylinder, an oblate and a prolate chamber at $\phi=30^{\circ}$ and with gravity. The spherical case produces a uniform circular bulge, of maximum amplitude $\simeq 1 \mathrm{~m}$, and about 7 times less than in 2D at this given internal overpressure. The associated plastic domain affects a relatively small area at the chambers' apex, in contrast with the cylindrical case where it is about to reach stage 3 . This shows that chamber overpressures produce about an order of magnitude differences in surface displacements depending on the chamber geometry.

A second series of tests displays the deformation field associated with an arbitrary maximum surface uplift of 1 meter, for four geometries at a correspondingly different overpressure (Fig. 10b). The spherical case requires $\Delta P=170 \mathrm{MPa}$ and shows maximum dilation at the surface. The cylindrical case requires only $30 \mathrm{MPa}$ and dilation at the surface has an amplitude of about half. An oblate chamber $(c=4 \mathrm{~km})$ requires $70 \mathrm{MPa}$, and maximum dilation occurs at the lateral tip at $7 \mathrm{~km}$ depth. A prolate chamber $(a=4$ $\mathrm{km}$ ) requires the greatest overpressure $\Delta P=240 \mathrm{MPa}$, and greatest dilation occurs at its apex. The large overpressure (> $100 \mathrm{MPa}$ ) required for both the spherical and prolate chambers to produce $1 \mathrm{~m}$ of surface uplift, leads to their failure at the apex. In contrast it is 'easier' (i.e. less overpressure) for the cylinder and oblate geometries to produce this 1 $\mathrm{m}$ of surface uplift, without generating any failure.

\subsection{Surface deformation and chamber geometry - Example Application}

Many volcanoes worldwide experience important surface uplift, without necessar-

ily evolving towards an eruption. An intriguing case is the Laguna del Maule in Southern Chile, which exhibits exorbitant inflation rates since the past ten years, accumulating a vertical displacement of 1.8 meters today and is still not erupting (Le Mevel et al , 2016). Using this as an example of a reservoir from which no magmatic conduit reaches the sur- 
face, we use our models to seek for a geometry and overpressure able to produce such surface uplift.

Fig. 10c-d shows that if the cause of this 1.8 meter uplift was an inflating prolate chamber, the contraction developing at the walls would impede the propagation of dikes. Moreover for both the spherical and prolate chambers, large overpressures are required to produce such uplift, pleading against such shapes being able to feed volcanic eruptions. Such geometries would rather explain the growth of a pluton supporting multiples injections of magma (increasing $\Delta P$ ), producing in-depth shear failure not reaching the surface.

The Maule volcanic complex includes past volcanic vents (Hildreth et al. 2010] forming a circular ring around the Maule central lake : this arrangement is consistent with a rather circular reservoir producing a conical failure geometry at depth, along which magmatic fluids may subsequently erupt to the surface [Mathieu et al. , 2008]. Such conical geometries are consistent with our 3D models of symmetrical oblate chambers. In our models prior to rupture at the walls, we showed (Fig. 6b) that an overpressure of $42 \mathrm{MPa}$ produces dilation at the tip that coincides with the location of maximum $\sigma_{3}$. This means that lateral dikes may nucleate there. In the Laguna del Maule many superficial low magnitude $(<2)$ seismic events were recorded 5 years after the onset of inflation, essentially in a peripherical area $5 \mathrm{~km}$ away from the center of inflation [Singer, 2014] and between 1 and $5 \mathrm{~km}$ depth, suggesting that indeed shear faults or dikes developed from the edges of the chamber (stage 2). However, in order for the plastic domain to connect all the way from the chamber to the surface, greater overpressure is required, $132 \mathrm{MPa}$ in our example Fig. 6d. This leads us to believe that the overpressure below the Laguna del Maule was so far insufficient to trigger an eruption.

Another information related to the Laguna del Maule, is that the last post-glacial eruptions display rhyolitic compositions [Hildreth et al., 2010], e.g. silicic and of relatively high viscosity. Following McLeod and Tait [1999] it is more difficult for high viscosity fluids to penetrate through the open cracks or dikes of a bedrock (because of frictional resistance), requiring high overpressure for a dike to propagate. Therefore, this may be another reason why the magma below the Laguna del Maule could not expel from its reservoir via lateral diking, consistent with the fact that this reservoir shape does not favor failure all the way from the chamber to the surface. This could explain why this volcanic complex has been inactive for 2.2 Myrs. 
Further detailed data analysis combined with numerical modeling are in progress

in order to propose a more accurate evolution of the Laguna del Maule volcanic system (Novoa Lizama et al., in prep).

\section{4 mode I or mode II failure and magma pathways}

In nature, both mode I and mode II magma pathways are observed in a variety of contexts, e.g. Woodcock and Underhill [1987]; White et al. [2011]; Plateaux et al. [2012];

Spacapan et al [2017]. For instance White et al. [2011] recorded seismically the propagation of a dike in Iceland over several months, via successive shearing-opening pistonlike pulses. How exactly the magma propagates through open conduits remains an open question, as this involves a variety of processes such as material embrittlement, mixing of heterogeneous viscous material, and reactive flow [Rubin, 1995; Petford et al., 2000; Townsend et al., 2015; Spacapan et al, 2017]. Our study focusses instead on the initiation of the conduit itself (our models are in the limit of the small strain continuum approximation).

By testing numerically whether mode I or mode II failure initiates and propagates from an overpressurised elliptical reservoir in two dimensions, Gerbault [2012a] related these mechanisms to two distinct conceptual views of the topology of the magma-rock wall interface (Rubin [1993] Fig.2). A first view-point, the one adopted here, supposes that the material at the wall of the magma chamber is 'intact', and then the local stress balance involves the (gravity-dependent) resistance of the hoop stress. This is the hypothesis adopted here, consistent with elasto-plastic behaviour at the crustal scale. The other conceptual view considers that the bedrock interface contains a multitude of microcracks so that at least one presents a favorable orientation allowing for the normal resisting lithostatic component of the bedrock to be cancelled out by the opposing lithostatic component of the internal fluid [Rubin, 1993]. Then hydrofracturing can occur at values close to the bedrock's tensile strength (Rubin [1993, 1995]; McTigue [1987]). Analogue models of liquid injection into gelatin validate this process and produce various shapes of coalesced liquid-filled buoyancy-driven pressure cracks [Takada, 1994; McLeod and Tait , 1999; Menand, 2011; Taisne et al. , 2011]. However note that these experiments imply an additional parameter, the buoyancy of the intruding fluid. 
A way to bridge mode I versus mode II fracture propagation is indeed to consider the pore-space on either side of the wall interface [Rozhko et al., 2007; Lucantonio et al. 2015; Keller et al. , 2013], in which case seepage processes involved in the local force balance control the macro-scale failure mode. Lucantonio et al. [2015] observed mode I crack propagation or arrest, at the interface of hydrogel layers of contrasting poro-elastic rigidity (and of identical density) depending on the regional stress field. The experimental works by Abdelmalak et al. [2012] and Mourgues et al. [2011] employing silicon injection in granular material and variable pore pressures, also showed a variety of geometrical evolutions of mode I and II propagating cracks, depending on the proportion of local versus regional pore-pressure.

Our models here do not account for poro-elasticity, but display domains of (mostly elastic) dilation of the order of $10^{-4}-10^{-3}$ over several cubic kilometers around the tips of oblate chambers (red areas in mid panels Figs. 7, 6, 8). These values of macroscopic volumetric expansion fall within a common range of processes of fluid mobilisation through crustal rocks (according to data from pressurized fracture experiments, stress oscillations experiments, natural springs or well temperatures, Manga et al. [2012]). Such volume increase likely generates microcracks along grain joints at the micro-scale, increasing the micro-porosity and thus increasing the connectivity of the porous medium. This increased connectivity may then enable hydrodynamic infiltration of magma through the bedrock over length scales significantly greater than the grain scale, provided magmatic fluids have a small enough viscosity and that they migrate faster than diffusive cooling [Rubin, 1995; Petford et al., 2000; Degruyter and Huber, 2014]. Several other strength weakening processes may further amplify the effective porosity of this connected medium, such as dissolution/crystallisation reactions between melts and bedrock [Kelemen et al. , 1995] leading to the transient formation and destruction of porous channels, and which over long time scales and length scales may result in diffuse flow of melt towards the surface [Kelemen et al. , 1995]. The connection of the magmatic fluids with the hydrothermal system via reaction-induced volume changes in the pore space [Delaney, 1982; Petford et al., 2000; Ingebritsen and Gleeson, 2015; Gardien et al., 2016] may also lead to (catastrophic) hydrodynamic connection up to the surface.

These informations lead us to propose that the dilational strain developping at the edges of our oblate chambers may well 1) enhance the bedrock's effective porosity and reduce its failure threshold, and therefore 2) potentially host magmas migrating out of the 
inflating reservoir. This proposition is coherent with other descriptions of magma flow through the crust; when considering the feeding of crustal plutons, Weinberg [1999] envisaged large dikes conduits, numerous narrow swarms, and the pervasive İD̆flow of channelled magma. Petford et al. [2000] described processes of magma ascent in the crust, either channelled along self-propagating dikes or pre-existing shear zones, but also within an "interconnected network of active shear zones and dilational structures". Given the high viscosity of crustal melts, these authors highlight the necessary role of matrix deformation, which is what our models reveal here.

We conclude that in the domain surrounding an inflating magma chamber, concurrent mechanisms of magma migration may occur, via percolation through the 'activated' porous medium in dilating 'pockets' at the chamber edges on the one hand, and along mode II fault conduits forming near and above the chamber's apex on the other hand. With increasing overpressure, the magma may ultimately reach the surface via a combination of both mechanisms and diking (mode I).

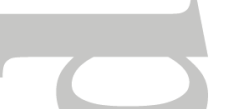

\subsection{Complementary thermo-mechanical effects}

Heat transport, magma injection rate, chemical differentiation, tectonic forcing, edifice loading and mechanical heterogeneities all contribute to a first order in bedrock microfracturation and macroscopic deformation. Below, we review the influence of other mechanical parameters, which basically add a stress component to the overall volcanic system, and which therefore mainly shifts the magnitude and location of the stress threshold enabling bedrock failure :

- Variations in the elastic and plastic properties throughout the bedrock such as the presence of weak layers or weak fault zones, are known to enable the relaxation and rotation of the stress field, which affects the geometries of diking and faulting [Gudmundsson, 1988, 2006; Masterlark, 2007; Rivalta et al., 2015; Karaoglu et al. 2016]. Similarly, a local increase in bedrock permeability such as by meteoritic fluids diminishes the bedrock strength, rotates the stress field and favor its failure [Plateaux et al., 2012].

- Regional tectonic stresses also modulate the pressure threshold for bedrock failure around a magma chamber. Tectonic extension favors dike emplacement by lowering the effective normal stress and thus the failure stress threshold (eg. Buck et al. 
[2006]; Gudmundsson [2006]; Grosfils [2007]); structurally, horizontal $\sigma_{3}$ favors vertical diking and normal faulting. In contrast, tectonic compression contributes to increasing the internal overpressure, but also increases the failure threshold (eg. Watanabe et al. [2002]; Galland et al. [2015]), with horizontal $\sigma_{1}$ favoring sill emplacement. Actually, volcanic activity does not always align according to the regional stress field, as for example along the tectono-volcanic Liquine-Ofzqui arc fault zone spanning along Southern Chile [Cembrano and Lara, 2009; Iturrieta et al. , 2016]. There, volcanic eruptions are not systematically triggered by great subduction earthquakes, although this is a mechanism often invoked to generate a tensile component in this tectono-volcanic fault zone [Bonali et al., 2013].

- The load exerted by an edifice adds a compressional component favoring lateral propagation of "fluid-filled cracks", as shown by analog [Takada, 1994; Watanabe et al. , 2002; Rivalta et al., 2015] and numerical work [Pinel and Jaupart, 2005; Corbi et al., 2015]. However Corbi et al. [2015] for instance fit surface failure structures (radial faults and circumferencial dikes) by considering only the gravitational load of the edifice itself, and neglect the gravity force acting on the entire medium. Given our previous remarks (see also discussion in Grosfils et al. [2015]), we would recommend to perform additional simulatioins that include the gravity force, and seek how to fit such failure patterns by integrating the whole temporal cycles of edifice building and caldera formation. Note that Currenti and Williams [2014] account for gravity and show that the presence of an edifice increases the critical overpressure capable to generate chamber failure, independent on the reservoir's geometry.

Nevertheless, the geophysical and geodetic record shows that magma chambers may not rupture at well-defined pressure values (e.g. Buck et al. [2006]; Segall [2013]). Worldwide statistical data [Manga et al. , 2012] indicate that many volcanic systems evolve at their proper "rythm", relatively independent from the regional stress field.

The buoyancy of magmatic fluids adds a driving force for upward magma migration, via hydrofracturing (e.g.Lister and Kerr [1991]; McLeod and Tait [1999]; Taisne et al. [2011]; Menand [2011]), shear failure (Keller et al. [2013]; Galland et al. [2015]) or fluid infiltration through the porous medium (e.g. Ingebritsen and Gleeson [2015]; Gardien et al. [2016]). In addition, magma differentiation within the chamber may generate 
a non-homogeneous density distribution along the chamber walls, with excess overpres-

sure occuring at the apex of the chamber where volatiles accumulate in contrast to deeper domains where dense material concentrates [Degruyter and Huber, 2014; Gelman et al., 2013]). Accounting for such vertical variations in mass distribution, in combination with variations in the reservoir's geometry should help improving the fit to geodetic observations.

In many arc volcanic systems, felsic magmas are erupted from a large central stratovolcano, while along the flanks more mafic compositions erupt with increasing distance from the center. Pinel and Jaupart [2005] have shown that the volcanic edifice controls the development of distal mafic dikes, which initiate from deeper levels of the reservoir where magmatic fluids are less differentiated. In our simulations, that do not take a volcanic edifice into account, failure patterns around oblate chambers show that shear failure occurs at the apex, while simultaneously tensile failure can develop at the lateral edges. We thus envision that magma flowing through shear conduits near the apex undergoes more frictional resistance and thus more differentiation over time, in contrast to less-viscous magma being able to flow out from deep lateral sources into the dilated pore space of the bedrock. Our models of oblate chambers might thus be good candidates for triggering both felsic central eruptions and distal more mafic eruptions.

Gas exsolution in the magma chamber may enhance mode I failure and trigger explosive eruptions (Ingebritsen et al. [2010]), whereas diffusive cooling can lead to either 'mushification' of the bedrock-magma melange [Degruyter and Huber, 2014], or viscous indentation of the bedrock [Spacapan et al, 2017]. The rheology of the bedrock via heat diffusion and advection processses thus introduces a time-dependent response of the bedrock [Jellinek and de Paolo , 2003; Karlstrom et al. , 2010] which is often recorded by geodetic data (for instance at the Laguna del Maule, Le Mevel et al. [2016]). Along this line, [Degruyter et al., 2016] argue that different types of eruptions at Santorini, Greece, witness magma transport processes evolving over different scales.

\section{Conclusions}

We studied the dynamics of bedrock shear failure around an inflating magmatic chamber (reservoir) in three-dimensions. We have evaluated the critical overpressure required for chamber wall failure (stage 1), benchmarked our numerical results against ana- 
lytical solutions, and studied the geometrical connection of the plastic domain between the chamber and the surface (stage 3). We have also compared surface deformation and the plastic state of the bedrock, both at given applied overpressure and at given surface uplift. Critical overpressures for the onset of shear failure at the chamber walls (stage 1) are proportional to the overburden column weight, unless either the friction angle is set to zero (approximating a weakened bedrock) or gravity is set to zero (approximating a bedrock at lithostatic pore-fluid pressure), in which cases the critical overpressure remains on the order of the tensile strength. These values provide upper and lower bounds respectively, for assessing the critical overpressure related to surface deformation above volcanic systems.

Even in a homogeneous bedrock, an inflating magma reservoir generates a heterogeneous stress field with rotations of $90^{\circ}$ in all directions. The observation of tensile structures above a volcanic system may thus indicate significant inflation of the underlying reservoir. Brittle plastic deformation between the reservoir walls at depth and the surface localises only in cases of sufficiently elongated oblate chambers. At the surface, localised excentric shear bands develop only at significant overpressure beyond the connection stage 3, whereas a ring-shaped domain of plastic strain (e.g. concentric shear faults) may form above prolate chambers during the intermediate stage 2 . These results may help identifying the geometry of deeper magma reservoirs from uplift data and field structures.

Our simulations show how 3D shapes of inflating magmatic chambers induce specific dilational elastic stress and strain patterns in the surrounding bedrock, which may thus enhance the connectivity of the pore space adjacent to the chamber walls, and enable 'diffuse' pathways for magma towards the surface. This is an alternative to the view of magma flowing inside localised dike conduits and shear zones departing from the chamber wall to the surface. Future models that account for both tensile failure and flow in the porous medium are required to predict how such diffuse or localised magma pathways evolve to trigger an eruption. Since the tectonic context, thermal effects, chemical differentiation, and frequency of magma injection also play primary roles, additional constraints from specific type localities are required to properly assess the dominant factors controling the stability of a volcanic system.

\section{Acknowledgments}

Souche's position is funded by the Research Council of Norway through the DIPS project (grant no. 240467). Michel Rabinowicz is thanked for thoughtful remarks on the ms., 
and M. de St Blanquat for sharing information on the Torres del Paine. The models were obtained thanks to the OMP community Cluster Nuwa (http://www.aero.obs-mip.fr/parcinstru/platmod). A-M. Cousin helped with figure edition. Two anonymous reviewers brought extended and constructive reviews that significantly improved this contribution. Thorsten Becker and Boris Kaus are thanked for watchful edition.

\section{References}

Abdelmalak, M. M., Mourgues, R., Galland, O., and Bureau, D. (2012). Fracture mode analysis and related surface deformation during dike intrusion: Results from 2D experimental modelling. Earth Planet. Sci. Lett. 359, 93-105.

Anderson, E. M. (1936), The dynamics of formation of cone sheets, ring dykes and cauldron subsidences, Proc. R. Soc. Edinburgh 56, 128-163.

Buck, W. R., Einarsson, P., BrandsdÃşttir, B. (2006). Tectonic stress and magma chamber size as controls on dike propagation: Constraints from the 1975-1984 Krafla rifting episode. J. Geophys. Res. 111, doi: 10.1029/2005JB003879.

Bonali, F. L., Tibaldi, A., Corazzato, C., Tormey, D. R., Lara, L. E. (2013). Quantifying the effect of large earthquakes in promoting eruptions due to stress changes on magma pathway: the Chile case. Tectonophysics, 583, 54-67.

Bonali, F. L., Tibaldi, A., Corazzato, C. (2015). Sensitivity analysis of earthquake-induced static stress changes on volcanoes: the $2010 \mathrm{Mw} 8.8$ Chile earthquake. Geophys. J. Int. 201(3), 1868-1890.

Burov, E., Jaupart, C., and GuillouâĂŘ-Frottier, L. (2003). Ascent and emplacement of buoyant magma bodies in brittleâĂ ̌̌-ductile upper crust. J. Geophys. Res. 108(B4).

Cembrano, J., Lara, L. (2009). The link between volcanism and tectonics in the southern volcanic zone of the Chilean Andes: a review. Tectonophysics, 471(1), 96-113.

Cerpa, N. G., Araya, R., Gerbault, M., Hassani, R. (2015). Relationship between slab dip and topography segmentation in an oblique subduction zone: Insights from numerical modeling. Geophys. Res. Lett. 42(14), 5786-5795.

Chéry, J., A. Bonneville, J. P. Villote, and D. Yuen (1991), Numerical modelling of caldera dynamical behaviour, Geophys. J. Int. 105, 365-379, doi:10.1111/j.1365246X.1991.tb06719.x.

Chéry, J., Zoback, M. D., Hassani, R. (2001). An integrated mechanical model of the San Andreas fault in central and northern California. J. Geophys. Res. 106(B10), 22051- 
22066.

Contreras M., Tassara A., Gerbault M., Araya R., Bataille K. (2016). Deformación intersÃysmica en zonas de subducción investigada por modelos numÃllricos 2D: estudio de caso antes del terremoto del Maule 2010. Andean geology, 43(3), 247-262.

Corbi, F., Rivalta, E., Pinel, V., Maccaferri, F., Bagnardi, M., Acocella, V. (2015). How caldera collapse shapes the shallow emplacement and transfer of magma in active volcanoes. Earth Planet. Sci. Lett. 431, 287-293.

Currenti, G., and Williams, C. A. (2014). Numerical modeling of deformation and stress fields around a magma chamber: Constraints on failure conditions and rheology.

Physics Earth Planet. Int. 226, 14-27.

Cundall, P. A. (1989), Numerical experiments on localization in frictional materials, Arch. Appl. Mech. 59, 148-159, doi:10.1007/BF00538368.

Cundall, P., and M. Board (1988). A microcomputer program for modeling largestrain plasticity problems, in Numerical Methods in Geomechanics, 2102-2108, A. A. Balkema, Rotterdam, Netherlands.

Degruyter W., and C. Huber (2014). A model for eruption frequency of upper crustal silicic magma chambers, Earth Planet. Sci. Lett. 403,117-130.

Degruyter, W., Huber, C., Bachmann, O., Cooper, K. M., Kent, A. J. (2016). Magma reservoir response to transient recharge events: The case of Santorini volcano (Greece). Geology, 44(1), 23-26.

Delaney, P. T. (1982). Rapid intrusion of magma into wet rock: groundwater flow due to pore pressure increases. J. Geophys. Res. 87(B9), 7739-7756.

Dieterich, J. H., Decker, R. W. (1975). Finite element modeling of surface deformation associated with volcanism. J. Geophys. Res. 80(29), 4094-4102.

Ernst, R. E., Head, J. W., Parfitt, E., Grosfils, E., Wilson, L. (1995). Giant radiating dyke swarms on Earth and Venus. Earth-Science Reviews, 39(1-2), 1-58.

d'Escatha, Y., and J. Mandel (1974), Stabilité d'une galerie peu profonde en terrain meuble, Extrait Ind. Miner., Num. Spec., 45-53.

Galland, O., Holohan, E., de Vries, B. V. W., Burchardt, S. (2015). Laboratory modelling of volcano plumbing systems: a review. In: Advances in Volcanology. Springer, Berlin, Heidelberg. doi: 0.1007/11157_2015_9.

Gardien, V., Rabinowicz, M., Vigneresse, J. L., Dubois, M., Boulvais, P., Martini, R. (2016). Long-lived interaction between hydrothermal and magmatic fluids in the Soultz- 
sous-ForÃłts granitic system (Rhine Graben, France). Lithos, 246, 110-127.

Gelman, S. E., Gutierrez, F. J., and Bachmann, O. (2013). On the longevity of large upper crustal silicic magma reservoirs. Geology, 41(7), 759-762.

Gerbault, M., A. Poliakov, and M. Daignières (1998), Prediction of faulting from the theories of elasticity and plasticity: What are the limits? J. Struct. Geol. 20, 301-320, doi:10.1016/S0191-8141(97)00089-8.

Gerbault, M., (2012). Pressure conditions for shear and tensile failure around a circular magma chamber; insight from elasto-plastic modelling Geol. Soc. London, Spec. Pub. 367-1, 111-130.

Gerbault, M., F. Cappa, and R. Hassani (2012). Elasto-plastic and hydromechanical models of failure around an infinitely long magma chamber Geochem. Geophys. Geosys. 136-3, doi:10.1029/2011GC003917.

Gregg, P. M., De Silva, S. L., Grosfils, E. B., and Parmigiani, J. P. (2012). Catastrophic caldera-forming eruptions: Thermomechanics and implications for eruption triggering and maximum caldera dimensions on Earth. J. Volc. Geoth. Res., 241, 1-12.

Grosfils, E. B. (2007), Magma reservoir failure on the terrestrial planets: Assessing the importance of gravitational loading in simple elastic models, J. Volcanol. Geoth. Res. 166, 47-75, doi:10.1016/j.jvolgeores.2007.06.007.

Grosfils, E. B., McGovern, P. J., Gregg, P. M., Galgana, G. A., Hurwitz, D. M., Long, S. M., Chestler, S. R. (2015). Elastic models of magma reservoir mechanics: a key tool for investigating planetary volcanism. Geol. Soc. London, Spec. Pub., 401(1), 239-267.

Gudmundsson, A. (1988), Effect of tensile stress concentration around magma chambers on intrusion and extrusion frequencies, J. Volc. Geotherm. Res. 35, 179-194.

Gudmundsson, A. (2006). How local stresses control magma-chamber ruptures, dyke injections, and eruptions in composite volcanoes. Earth Sci. Reviews 79(1), 1-31.

Gudmundsson, A. (2007). Gudmundsson, A. (2007). Conceptual and numerical models of ring-fault formation. J. Volcan. Geoth. Res. 164(3), 142-160.

Hassani, R., Chéry, J. (1996). Anelasticity explains topography associated with Basin and Range normal faulting. Geology, 24(12), 1095-1098.

Hassani, R., D. Jongmans, and J. Chéry, (1997). Study of plate deformation and stress in subduction processes using two-dimensional numerical models, J. Geophys. Res., 102, 17951-17965. 
Haug, Ã Ÿ. T., Galland, O., Souloumiac, P., Souche, A., Guldstrand, F., Schmiedel, T. (2017). Inelastic damage as a mechanical precursor for the emplacement of saucershaped intrusions. Geology, 45(12), 1099-1102.

He, J., Chery, J. (2008). Slip rates of the Altyn Tagh, Kunlun and Karakorum faults (Tibet) from 3D mechanical modeling. Earth Planet. Sci. Lett., 274(1), 50-58.

Hildreth, W., Godoy, E., Fierstein, J., Singer, B. (2010). Laguna del Maule Volcanic field: eruptive history of a Quaternary basalt-to-rhyolite distributed volcanic field on the Andean range crest in central Chile. Servicio Nacional de Geología y Minería-Chile Boletin, 63, 142.

Hubbert, M. K., Rubey, W. W. (1959). Role of fluid pressure in mechanics of overthrust faulting I. Mechanics of fluid-filled porous solids and its application to overthrust faulting. Geol. Soc. Am. Bull, 70(2), 115-166.

Ikari, M. J., Saffer, D. M., Marone, C. (2009). Frictional and hydrologic properties of clay-rich fault gouge. J. Geophys.Res. 114(B5).

Ingebritsen, S. E., Geiger, S., Hurwitz, S., Driesner, T. (2010). Numerical simulation of magmatic hydrothermal systems. Reviews of Geophysics, 48(1), doi:10.1111/gfl.12118. Ingebritsen, S. E., Gleeson, T. (2015). Crustal permeability: Introduction to the special issue. Geofluids, 15(1-2), 1-10.

Iturrieta, P. C., Hurtado, D. E., Cembrano, J., Stanton-Yonge, A. (2017). States of stress and slip partitioning in a continental scale strike-slip duplex: Tectonic and magmatic implications by means of finite element modeling. Earth Planet. Sci. Lett. 473, 71-82.

Jeffery, G.B., 1921. Plane stress and plane strain in bipolar coordinates. Phil. Trans. Royal Soc. London, Series A 221, 265-293.

Jellinek, A. M., and D. J. De Paolo (2003), A model for the origin of large silicic magma chambers: precursors of caldera-forming eruptions, Bull. Volcanol. 65, 363-381.

Karaoglu , O., Browning, J., Bazargan, M., and Gudmundsson, A. (2016). Numerical modelling of triple junction tectonics at Karliova, Eastern Turkey, with implications for regional magma transport. Earth Planet. Sci. Lett. 452, 157-170.

Karlstrom, L., Dufek, J., Manga, M. (2010). Magma chamber stability in arc and continental crust. J. Volcan. Geoth. Res. 190(3), 249-270.

Kelemen, P. B., Whitehead, J. A., Aharonov, E., Jordahl, K. A. (1995). Experiments on flow focusing in soluble porous media, with applications to melt extraction from the mantle. J. Geophys. Res. 100(B1), 475-496. 
Keller, T., May, D. A., Kaus, B. J. (2013). Numerical modelling of magma dynamics coupled to tectonic deformation of lithosphere and crust. Geophys. J. Int. 195(3), 14061442.

Le Mevel, H., Gregg, P. M., and Feigl, K. L. (2016). Magma injection into a long lived reservoir to explain geodetically measured uplift: Application to the 2007-2014 unrest episode at Laguna del Maule volcanic field, Chile. J. Geophys. Res. 121(8), 6092-6108.

Leuthold, J., MÃijntener, O., Baumgartner, L. P., Putlitz, B., Ovtcharova, M., Schaltegger, U. (2012). Time resolved construction of a bimodal laccolith (Torres del Paine, Patagonia). Earth Planet. Sci. Lett. 325, 85-92.

Lister, J. R., and Kerr, R. C. (1991). Fluid mechanical models of crack propagation and their application to magma transport in dykes.J. Geophys. Earth 96(B6), 10049-10077.

Lucantonio, A., Noselli, G., Trepat, X., DeSimone, A., Arroyo, M. (2015). Hydraulic fracture and toughening of a brittle layer bonded to a hydrogel. Physical Review letters, 115(18), 188105.

Manga, M., Beresnev, I., Brodsky, E. E., Elkhoury, J. E., Elsworth, D., Ingebritsen, S. E., Wang, C. Y. (2012). Changes in permeability caused by transient stresses: Field observations, experiments, and mechanisms. Reviews of Geophysics 50(2), doi: 10.1029/2011RG000382.

Masterlark, T. (2007). Magma intrusion and deformation predictions: Sensitivities to the Mogi assumptions. J. Geophys. Res., 112(B6), doi:10.1029/2006JB004860.

Mathieu, L., De Vries, B. V. W., Holohan, E. P., Troll, V. R. (2008). Dykes, cups, saucers and sills: Analogue experiments on magma intrusion into brittle rocks. Earth Planet. Sci. Lett. 271(1), 1-13.

McLeod, P., Tait, S. (1999). The growth of dykes from magma chambers. J. Volcan.

Geoth. Res. 92(3), 231-245.

McTigue, D. F. (1987). Elastic stress and deformation near a finite spherical magma body: resolution of the point source paradox. J. Geophys. Res. 92(B12), 12931-12940.

Menand, T. (2011). Physical controls and depth of emplacement of igneous bodies: A review. Tectonophysics, 500(1), 11-19.

Minakov, A., Yarushina, V., Faleide, J. I., Krupnova, N., Sakoulina, T., Dergunov, N., Glebovsky, V. (2017). Dyke emplacement and crustal structure within a continental large igneous province, northern Barents Sea. Geol. Soc London, Spec. Pub., 460, SP460-4. 
Mogi, K. (1958). Relationships between the eruptions of various volcanoes and the deformations of the ground surfaces around them. Bull. Earthquake Res. Inst., Univ. Tokyo 36, 99-134.

Mourgues, R., Gressier, J. B., Bodet, L., Bureau, D., Gay, A. (2011). 'Basin scale' versus 'localized' pore pressure/stress coupling - Implications for trap integrity evaluation. Marine Petrol. Geol., 28(5), 1111-1121.

Nádai A. (1931). Plasticity: Mechanics of the plastic state of matter. McGraw-Hill, 349 p.

Ortiz, M., Simo, J. C. (1986). An analysis of a new class of integration algorithms for elastoplastic constitutive relations. Int. J. Num. Meth. in Engineering, 23(3), 353-366.

Petford, N., Cruden, A. R., McCaffrey, K. J. W., Vigneresse, J. L. (2000). Granite magma formation, transport and emplacement in the Earth's crust. Nature, 408(6813), 669-673.

Pinel, V., Jaupart C. (2005), Caldera formation by magma withdrawal from a reservoir beneath a volcanic edifice, Earth Planet. Sci. Lett. 230, 273-287.

Plateaux, R., Bergerat, F., Béthoux, N., Villemin, T., Gerbault, M. (2012). Implications of fracturing mechanisms and fluid pressure on earthquakes and fault slip data in the east Iceland rift zone. Tectonophysics, 581, 19-34.

Poliakov, A. N. B., Cundall, P. A., Podladchikov, Y. Y., Lyakhovsky, V. A. (1993). An explicit inertial method for the simulation of viscoelastic flow: an evaluation of elastic effects on diapiric flow in two-and three-layers models. In Flow and Creep in the Solar System: observations, modeling and Theory (pp. 175-195). Springer Netherlands.

Remy, D., Froger, J. L., Perfettini, H., Bonvalot, S., Gabalda, G., Albino, F., Cayol V., Legrand D., de Saint-Blanquat M. (2014). Persistent uplift of the Lazufre volcanic complex (Central Andes): New insights from PCAIM inversion of InSAR time series and GPS data. Geochemistry, Geophysics, Geosystems, 15(9), 3591-3611.

Rivalta, E., Taisne, B., Bunger, A. P., Katz, R. F. (2015). A review of mechanical models of dike propagation: Schools of thought, results and future directions. Tectonophysics, $638,1-42$.

Rozhko, A. Y., Podladchikov, Y. Y., F. Renard (2007). Failure patterns caused by localized rise in poreâĂ $\breve{R}$-fluid overpressure and effective strength of rocks. Geophys. Res. Lett. $34(22)$.

Rubin, A. M. (1993). Dikes vs. diapirs in viscoelastic rock, Earth Planet Sci. Lett. 117, 653-670. 
Rubin, A. M. (1995). Propagation of magma-filled cracks, Annu. Rev. Earth Planet. Sci., 23, 287-336, doi:10.1146/annurev.ea.23.050195.001443.

Salençon, J. 1969. La théorie des charges limites dans la résolution des problấlmes de plasticité en déformation plane. PhD thesis, Laboratoire de Mécanique des Solides de l'Ecole Polytechnique, Paris.

Segall, P. (2013), Volcano deformation and eruption forecasting, Geol. Soc. London, Spec. Pub. 380.1, 85-106.

Singer, B. S., Andersen, N. L., Le MÃlvel, H., Feigl, K. L., DeMets, C., Tikoff, et al., (2014). Dynamics of a large, restless, rhyolitic magma system at Laguna del Maule, southern Andes, Chile. GSA Today, 24(12), 4-10.

Skarmeta, J. J., Castelli, J. C. (1997). Intrusión sintectónica del granito de las Torres del Paine, Andes Patagónicos de Chile. Andean Geology, 24(1), 55-74.

Spacapan, J. B., Galland, O., Leanza, H. A., Planke, S. (2017). Igneous sill and finger emplacement mechanism in shale-dominated formations: a field study at Cuesta del Chihuido, NeuquÃln Basin, Argentina. J. Geol. Soc., 174(3), 422-433.

Taisne, B., Tait, S., Jaupart, C. (2011). Conditions for the arrest of a vertical propagating dyke. Bulletin of Volcanology, 73(2), 191-204.

Tait, S., C. Jaupart, and S. Vergniolle (1989), Pressure, gas content and eruption periodicity of a shallow, crystallizing magma chamber, Earth Planet. Sci. Lett. 92, 107-123, doi:10.1016/0012-821X(89)90025-3.

Takada, A. (1994). Development of a subvolcanic structure by the interaction of liquidfilled cracks. J. Volcan. Geoth. Res., 61(3), 207-224.

Timoshenko, S., and J. N. Goodier (1970), Theory of Elasticity 2nd ed. 608 pp., McGrawHill, New York.

Townsend, M., Pollard, D. D., Johnson, K., Culha, C. (2015). Jointing around magmatic dikes as a precursor to the development of volcanic plugs. Bulletin of Volcanology, $77(10), 92$.

Vermeer, P. A., and R. de Borst (1984), Non-associated plasticity for soils, concrete and rocks, Heron, 29, 1-75.

Watanabe, T., Masuyama, T., Nagaoka, K., Tahara, T. (2002). Analog experiments on magma-filled cracks. Earth, planets and space, 54(12), 1247-1261.

Weinberg, R. F. (1999). Mesoscale pervasive felsic magma migration: alternatives to dyking. Lithos, 46(3), 393-410. 
White, R. S., Drew, J., Martens, H. R., Key, J., Soosalu, H., JakobsdÃ A̧ţtir, S. S. (2011).

Dynamics of dyke intrusion in the mid-crust of Iceland. Earth Planet. Sci. Lett. 304(3), 300-312.

Woodcock, N. H., Underhill, J. R. (1987). Emplacement-related fault patterns around the Northern Granite, Arran, Scotland. Geol. Soc. Am. Bull., 98(5), 515-527.
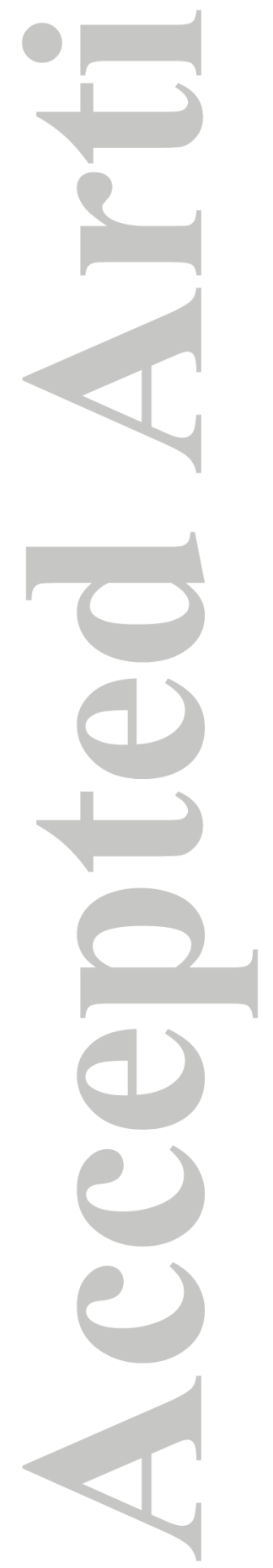
Table 1. Models information. Column 2 gives chamber depth $H$ and gravity (on $g$ ), off (0)? Next columns give axes

$a, b, c$ and friction $\phi$. Then the location of initial failure (InitF), the overpressure associated with wall failure $\left(\Delta P_{1}\right)$ and with connection of the plastic domain to the surface (Stage 3, $\Delta P_{3}$ ). Overpressures are obtained with a precision of $10 \%$. CW models refer to the geometries prescribed by Currenti and Williams [2014].

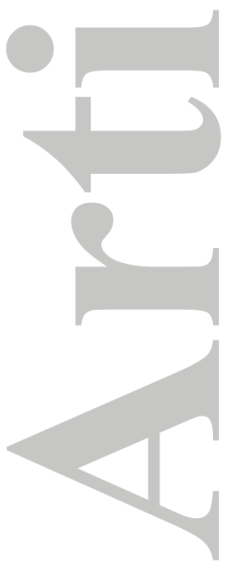

\begin{tabular}{|c|c|c|c|c|c|c|}
\hline Test & $\mathrm{H}$ & Ellipse axes(km) & $\phi, G$ & InitF & $\Delta P_{1}(\mathrm{MPa})$ & $\Delta P_{3}(\mathrm{MPa})$ \\
\hline cylinder & 7 & $b=2, c=\infty$ & 0,10 & wall & 13 & 29.5 \\
\hline sphere & 7 & $a=b=c=2$ & 0,10 & wall & 14 & 56 \\
\hline cylobl $/ 7 \mathrm{~km}$ & 7 & $b=7, c=\infty$ & 0,10 & latt & 7 & 12 \\
\hline symobl/4km & 7 & $b=c=4$ & 0,10 & latt & 11 & 35 \\
\hline symobl $/ 7 \mathrm{~km}$ & 7 & $b=c=7$ & 0,10 & surf-latt & 5.5 & 20 \\
\hline obl2/4km & 7 & $a=b=2, c=4$ & 0,10 & wall & 13 & 44 \\
\hline obl2/7km & 7 & $a=b=2, c=7$ & 0,10 & latt-wall & 12 & 38 \\
\hline obl2/20km & 7 & $a=b=2, c=20$ & 0,10 & latt & 12 & 33 \\
\hline pro $7 / 4 \mathrm{~km}$ & 7 & $a=4, b=c=2$ & 0,10 & apex & 11 & 44 \\
\hline pro $9 / 4 \mathrm{~km}$ & 9 & $a=4, b=c=2$ & 0,10 & apex & 13 & 56 \\
\hline pro12/7km & 12 & $a=7, b=c=2$ & 0,10 & apex & 12 & 58 \\
\hline cylinder & 7 & $b=2, c=\infty$ & 30,0 & surf & 12 & 32 \\
\hline sphere & 7 & $a=b=c=2$ & 30,0 & wall & 15 & 86 \\
\hline obl $2 / 4 \mathrm{~km}$ & 7 & $a=b=2, c=4$ & 30,0 & latt & 10 & 39 \\
\hline pro $9 / 2 \mathrm{~km}$ & 9 & $a=4, b=c=2$ & 30,0 & wall & 12 & 89 \\
\hline cylinder & 7 & $b=2, c=\infty$ & 30,10 & surf & 105 & 160 \\
\hline sphere & 7 & $a=b=c=2$ & 30,10 & wall & 120 & 420 \\
\hline cylobl $/ 7 \mathrm{~km}$ & 7 & $b=7, c=\infty$ & 30,10 & surf-latt & 25 & 60 \\
\hline symobl $/ 7 \mathrm{~km}$ & 7 & $b=c=2.4$ & 30,10 & surf-latt & 138 & 330 \\
\hline symobl/4km & 7 & $b=c=4$ & 30,10 & surf-latt & 87 & 198 \\
\hline symobl $/ 7 \mathrm{~km}$ & 7 & $b=c=7$ & 30,10 & surf-latt & 38 & 108 \\
\hline obl2/4km & 7 & $a=b=2, c=4$ & 30,10 & surf-latt & 120 & 280 \\
\hline obl2/7km & 7 & $a=b=2, c=7$ & 30,10 & surf-latt & 98 & 220 \\
\hline obl $2 / 20 \mathrm{~km}$ & 20 & $a=b=2, c=20$ & 30,10 & surf-latt & 85 & 175 \\
\hline pro $7 / 2 \mathrm{~km}$ & 7 & $a=4, b=c=2$ & 30,10 & apex & 56 & 201 \\
\hline pro $9 / 2 \mathrm{~km}$ & 9 & $a=4, b=c=2$ & 30,10 & apex & 90 & 480 \\
\hline pro12/7km & 12 & $a=7, b=c=2$ & 30,10 & apex & 84 & 539 \\
\hline $\mathrm{CW} 2 \mathrm{~km} \lambda 1$ & 2 & $a=b=c=0.5$ & 35,10 & apex & 45 & 215 \\
\hline CW $3.5 \mathrm{~km} \lambda 1$ & 3.5 & $a=b=c=0.5$ & 35,10 & apex & 85 & 810 \\
\hline CW $5 \mathrm{~km} \lambda 1$ & 5 & $a=b=c=0.5$ & 35,10 & apex & 120 & 1710 \\
\hline CW $2 \mathrm{~km} \lambda 0.2$ & 2 & $a=0.5, b=c=0.1$ & 35,10 & apex & 30 & 1012 \\
\hline $\mathrm{CW} 2 \mathrm{~km} \lambda 0.6$ & 2 & $a=0.5, b=c=0.3$ & 35,10 & apex & 32.5 & 390 \\
\hline $\mathrm{CW} 2 \mathrm{~km} \lambda 1.5$ & 2 & $a=0.5, b=c=0.75$ & 35,10 & apex & 42 & 123 \\
\hline $\mathrm{CW} 2 \mathrm{~km} \lambda 2$ & 2 & $a=0.5, b=c=1.0$ & 35,10 & surf-latt & 35 & 90 \\
\hline $\mathrm{CW} 2 \mathrm{~km} \lambda 2.5$ & 2 & $a=0.5, b=c=1.25$ & 35,10 & surf-latt & 30 & 80 \\
\hline $\mathrm{CW} 2 \mathrm{~km} \lambda 3$ & 2 & $a=0.5, b=c=1.5$ & 35,10 & surf-latt & 20 & 65 \\
\hline
\end{tabular}




\section{Figure Captions}

Figure 1. Numerical setup for an inflating magma chamber: model geometry and boundary conditions.

Figure 2. Cylindrical chambers, evolution of plastic domain with increasing overpressure $\Delta P$ using a radius $a=2 \mathrm{~km}$ and a depth $H=7 \mathrm{~km}$, similar to Gerbault et al. (2012). a) case with gravity and friction $\phi=0^{\circ}$, from left to right at 3 steps with $\Delta P=22,31,34$ MPa respectively. b) case with $\phi=30^{\circ}$ and no gravity ( $\mathrm{g}=0$ ), at $\Delta P=21,31,36 \mathrm{MPa}$. c) Case with gravity and $\phi=30$, at $\Delta P=125,185,225 \mathrm{MPa}$. d) Oblate case with gravity and $\phi=30^{\circ}$ at $\Delta P=35,50,60 \mathrm{MPa}$.

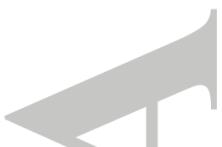

Figure 3. Comparison of overpressure $\Delta P$ necessary to initiate failure at the chamber wall with, a) variable spherical chamber depth, and b) variable chamber shape $\lambda=c / a$ (oblate chambers have $\lambda>1$ ). The analytical approximation by Gerbault [2012a] predicts twice the value of Eq.3. Red curves refer to Adeli models $(H=7 \mathrm{~km}, R=2 \mathrm{~km})$, blue curves correspond to $R=0.5 \mathrm{~km}$ as in Currenti and Williams [2014] (labelled "CW"). Complementary models by Souche are labelled "Adhoc".

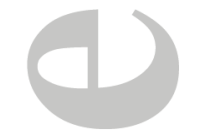

Figure 4. Spherical chambers $(a=2 \mathrm{~km})$ : plastic domains evolving with increasing overpressure from left to right for three cases: a) friction angle $\phi=0^{\circ}$ and gravity at $\left.\Delta P=24,54,58 \mathrm{MPa}, \mathrm{b}\right) \phi=30^{\circ}$ and no gravity at $\Delta P=20,58,90 \mathrm{MPa}$, and c) $\phi=30^{\circ}$ and gravity at $\Delta P=160,420,500 \mathrm{MPa}$.

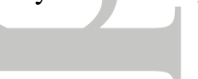

Figure 5. Comparison of plastic domains between cylindrical (a) and spherical (b-c) geometries, with radius $a=2 \mathrm{~km}$ and depth $H=7 \mathrm{~km}, \phi=30$ and gravity. (b) shows a stage prior to connection stage 3 , which is displayed in a) and c). From left to right for each case, the plastic shear strain $\left(\varepsilon_{p}\right)$, dilational strain $\left(\varepsilon_{I}\right)$, and principal stresses (magnitude of $\sigma_{3}$ as colour contours and $\sigma_{1}$ as line orientations).

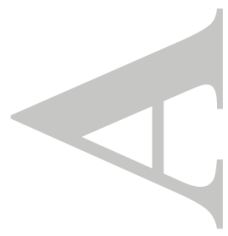


Figure 6. Oblate cylindrical and symmetrical chamber models, with depth $H=7 \mathrm{~km}$, vertical axis $a=2$ $\mathrm{km}$, friction angle $\phi=30^{\circ}$, and gravity. Cases a) cylinder with $b=7 \mathrm{~km}, \mathrm{~b}-\mathrm{c}$ ) Oblate $b=c=7 \mathrm{~km}$, and d) oblate $b=c=4 \mathrm{~km}$. From left to right: strains $\varepsilon_{p}, \varepsilon_{I}$, and $\sigma_{3}$ stress contours with $\sigma_{1}$ orientations. (a), (c), (d) display stage 3 when the plastic domain connects the chamber to the surface, whereas b) shows a stage previous to wall failure.

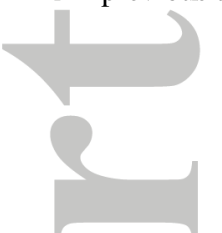

Figure 7. Asymmetric oblate chamber models. Depth $H=7 \mathrm{~km}, a=b=2 \mathrm{~km}$, friction $\phi=30$, when the plastic domain between the chamber and the surface is connected. a) $c=4 \mathrm{~km}, \mathrm{~b}) c=7 \mathrm{~km}, \mathrm{c}) c=20 \mathrm{~km}$.

From left to right: strains $\varepsilon_{p}, \varepsilon_{1}$, and principal stresses $\sigma_{3}$ contours together with $\sigma_{1}$ orientations.
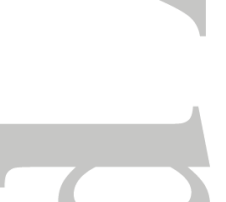

Figure 8. Prolate chambers, with $\phi=30^{\circ}$, axes $b=c=2 \mathrm{~km}$ and variable vertical axis $a$ and depth $H$ : a) during stage 1 with wall failure (see inner legend for values of $H, a$, gravity and $\Delta P$ ). b) Intermediate stage 2 for a model with $H=12, a=7 \mathrm{~km}$ and gravity (same as first column above), then c) at connection stage 3, showing from left to right: plastic strain $\varepsilon_{p}$, first strain invariant $\varepsilon_{1}$, and principal stresses with $\sigma_{3}$ colours and $\sigma_{1}$ orientations.

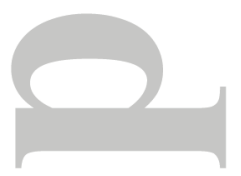

Figure 9. Cases with radially expanding ('excentric') shear bands at the surface, well after connection stage 3. a) Oblate chamber with gravity at $\Delta P=360 \mathrm{MPa}$. b) Spherical chamber without gravity at $\Delta P=120 \mathrm{MPa}$. In both cases friction angle $\phi=30^{\circ}$.

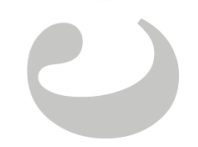

Figure 10. Models at a given applied overpressure $\Delta P=150 \mathrm{MPa}$ (with gravity and $\phi=30^{\circ}$ ): a) Surface displacements (uplift $U z$ and horizontal $U x$ ) and b) plastic strain, for a sphere, a cylinder, an oblate and a prolate chamber. Models producing a maximum surface uplift of 1 meter for these 4 different geometries: c) Surface displacements, d)plastic strain (mid panel) and first strain invariant (e). 


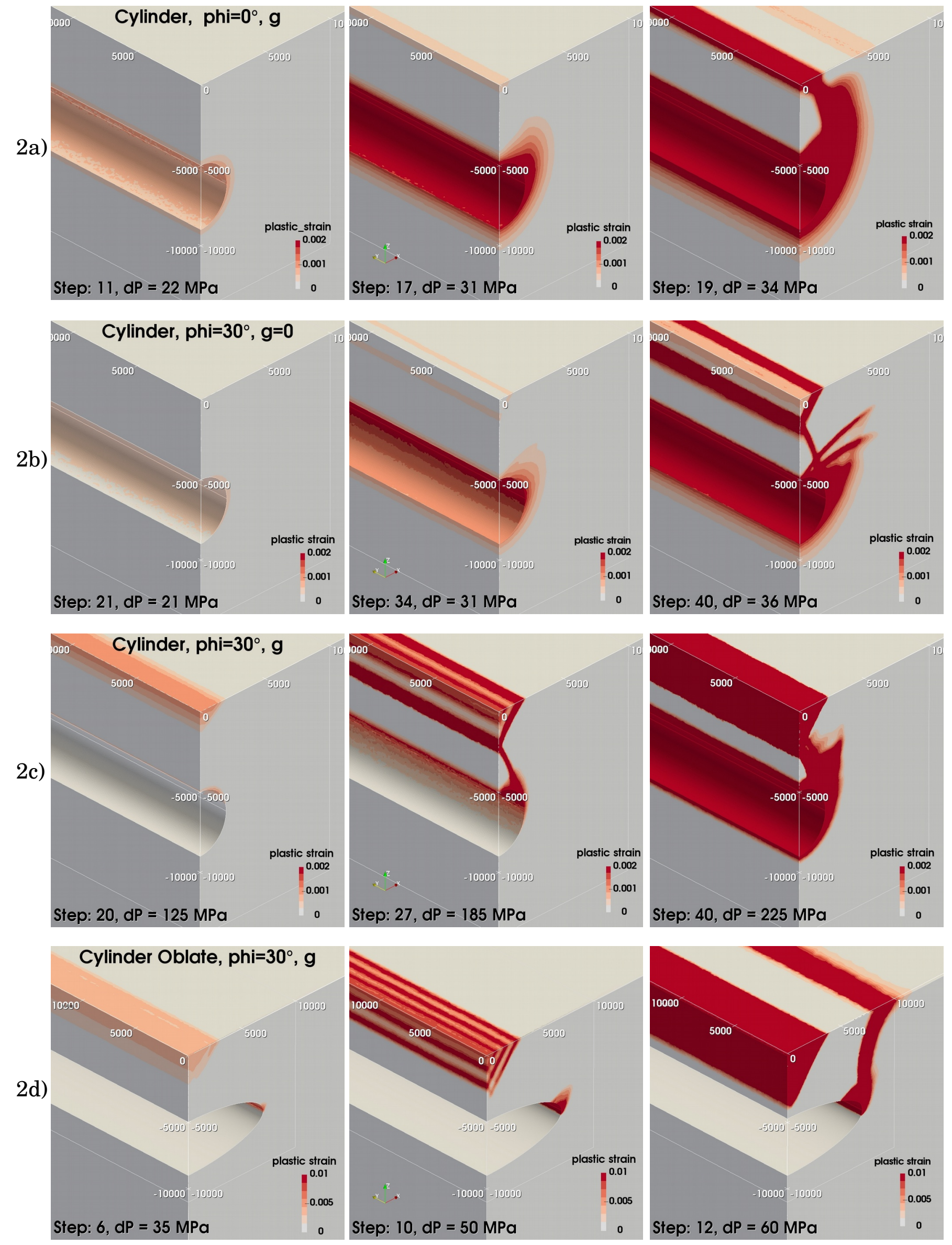

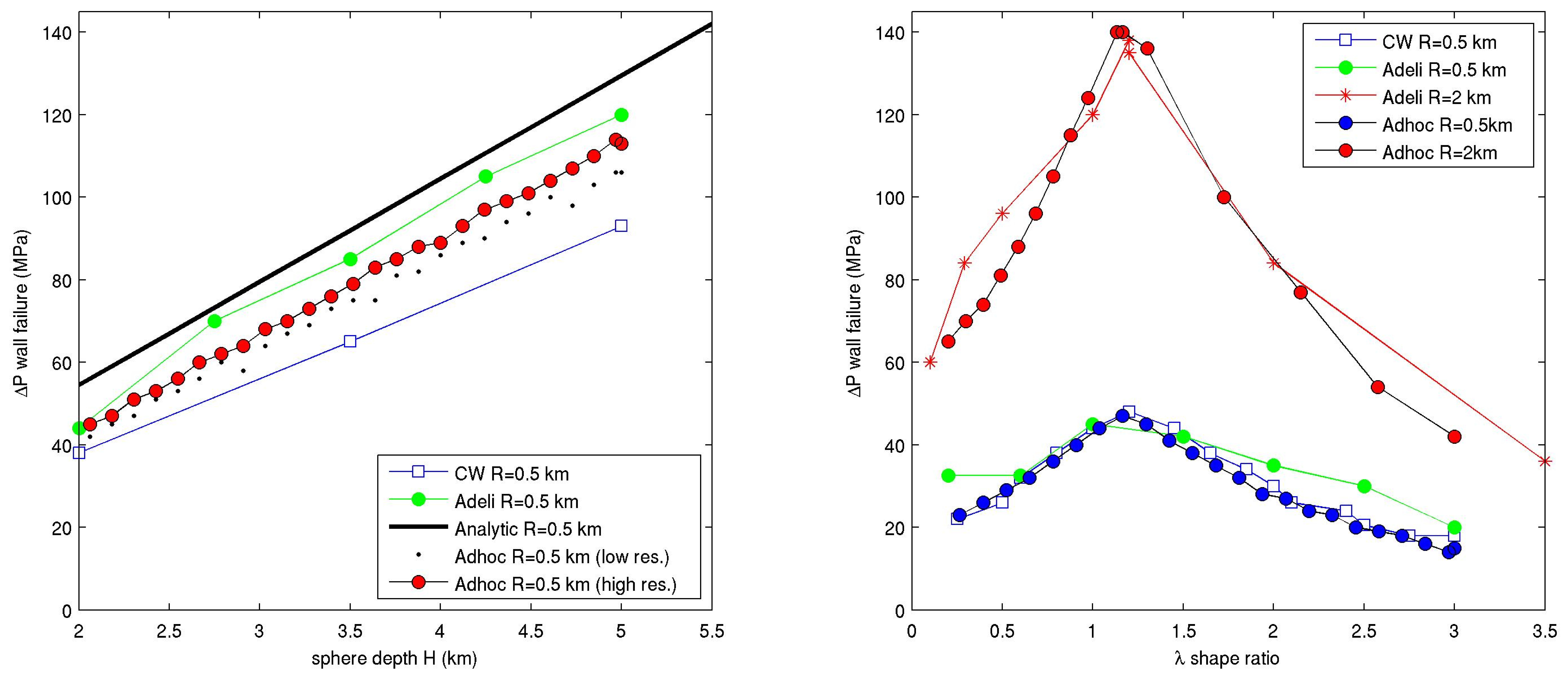
4a)
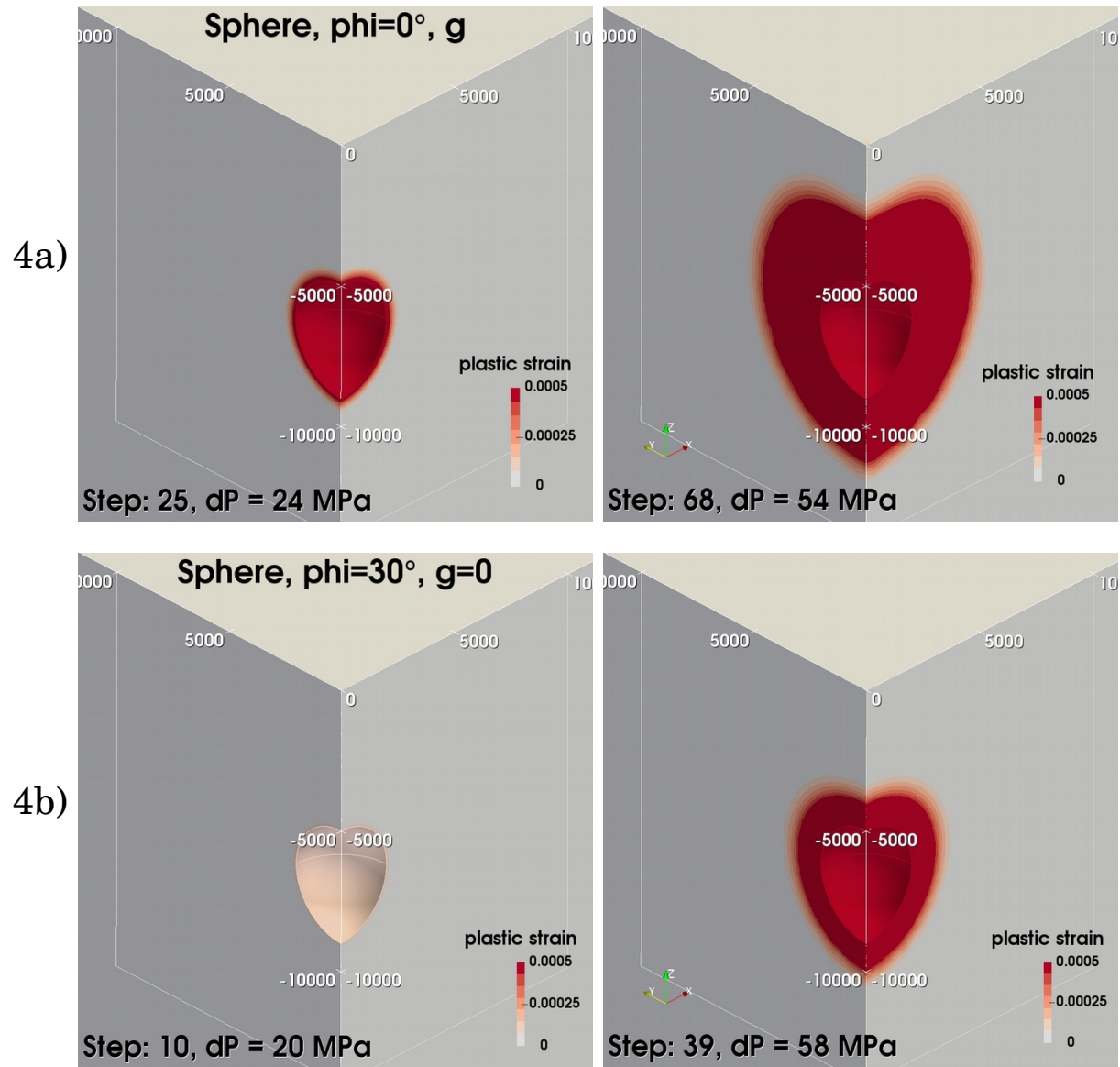

\section{Sphere, $\mathrm{phi}=30^{\circ}, \mathrm{g}$}

4c)

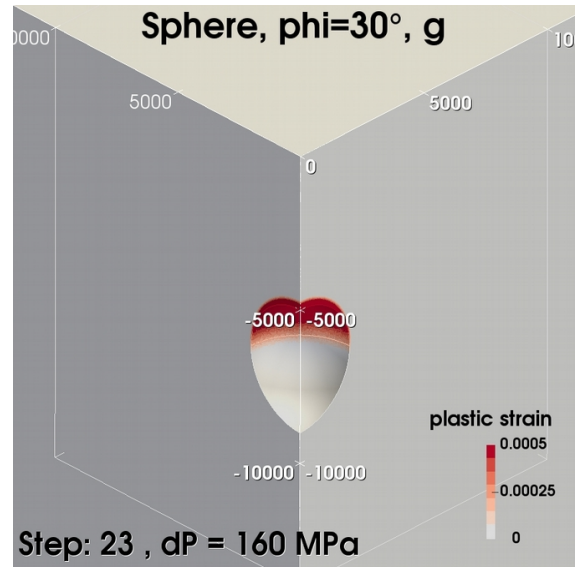

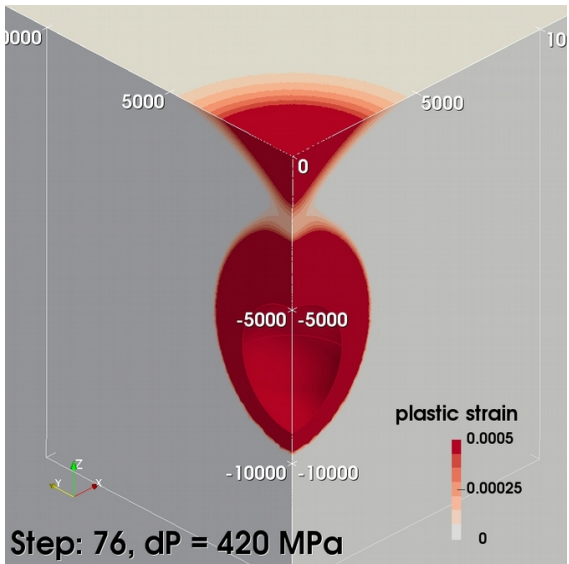
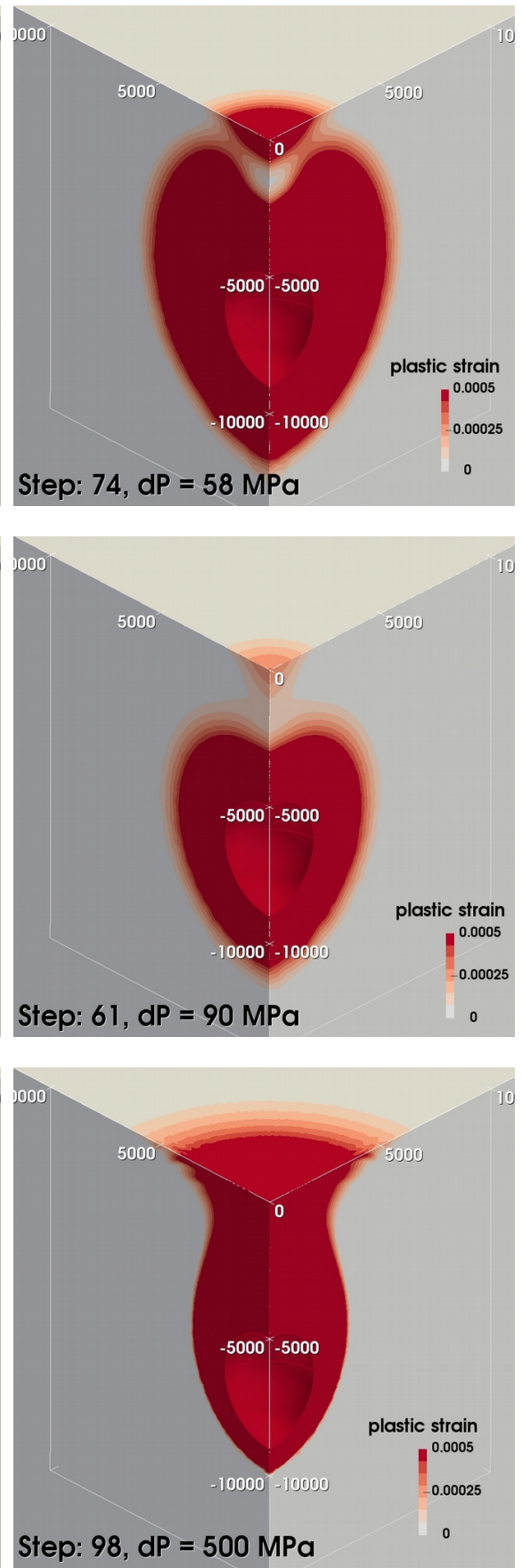
5a)

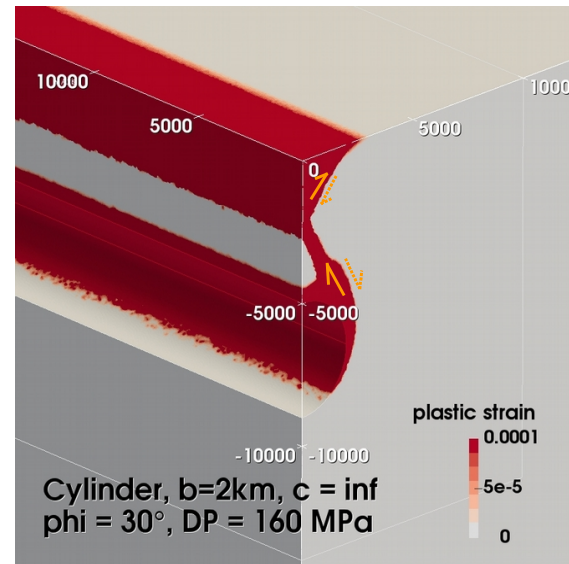

5b)
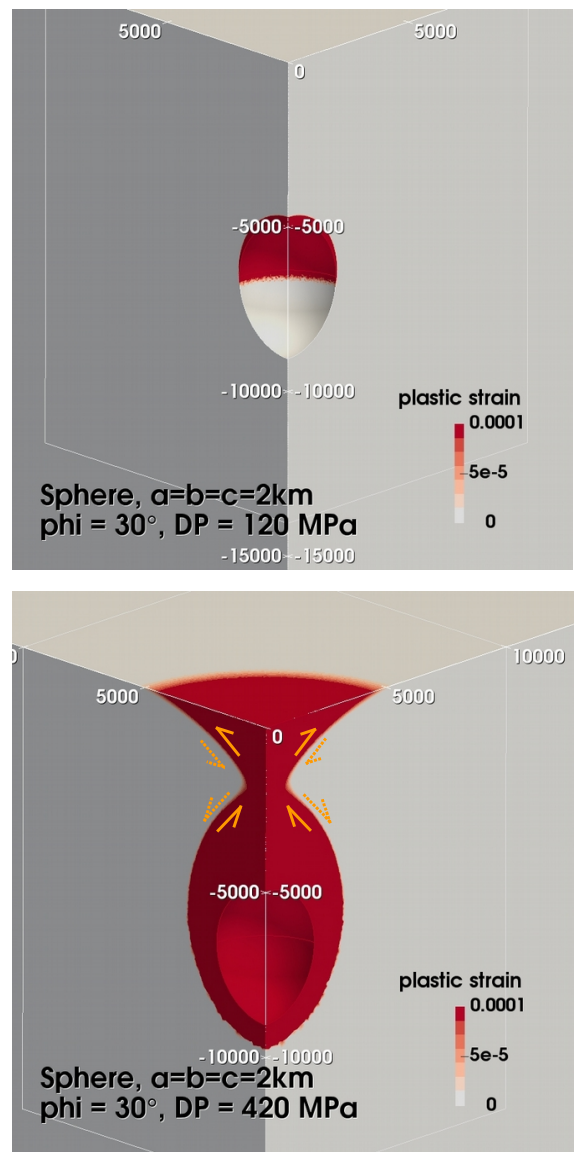
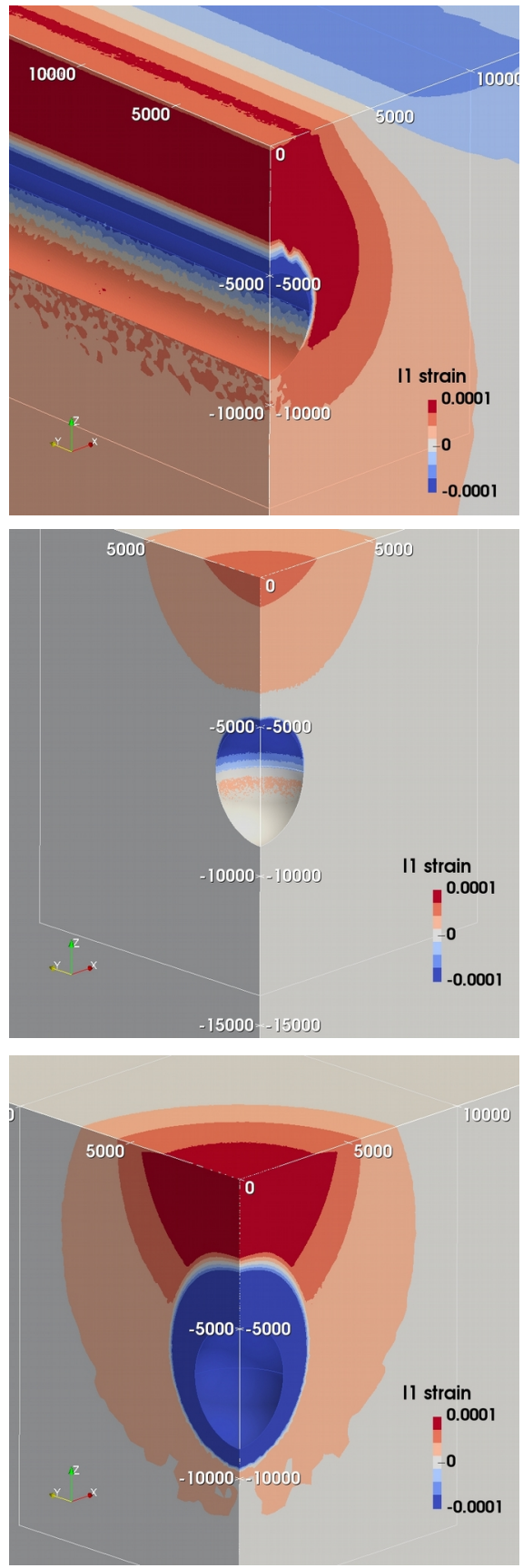
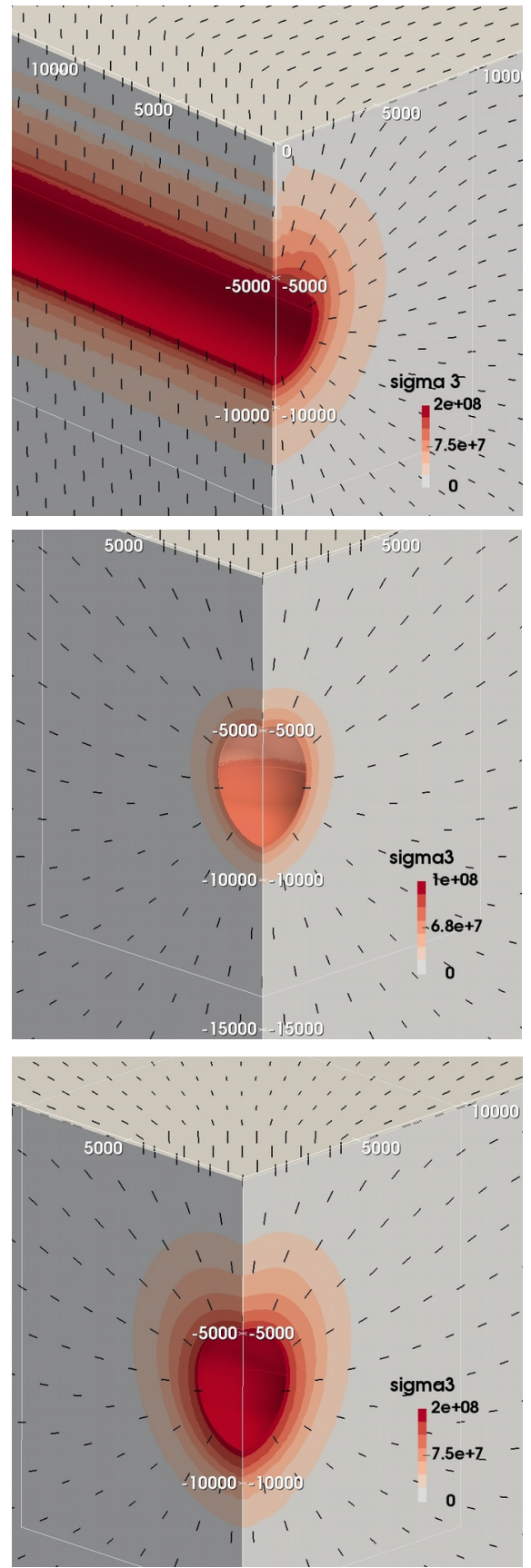
6a)

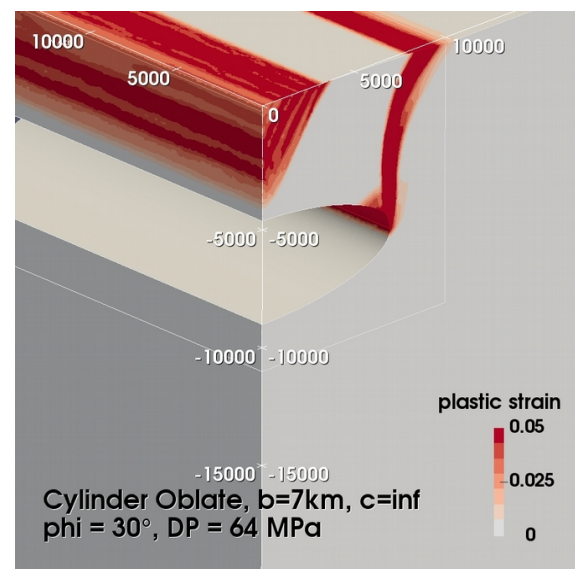

6b)

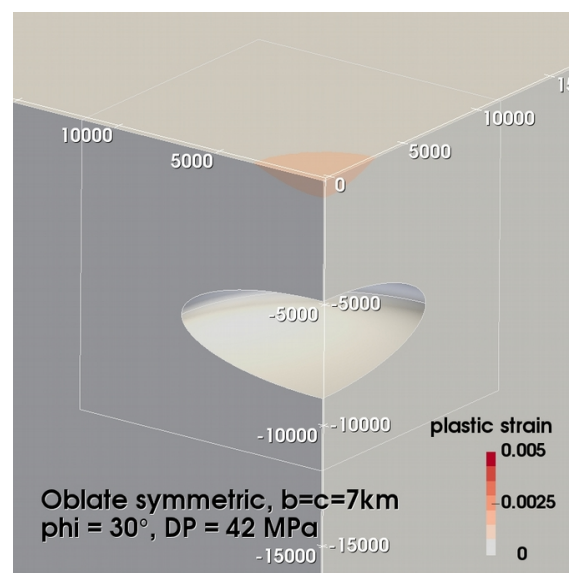

6c)
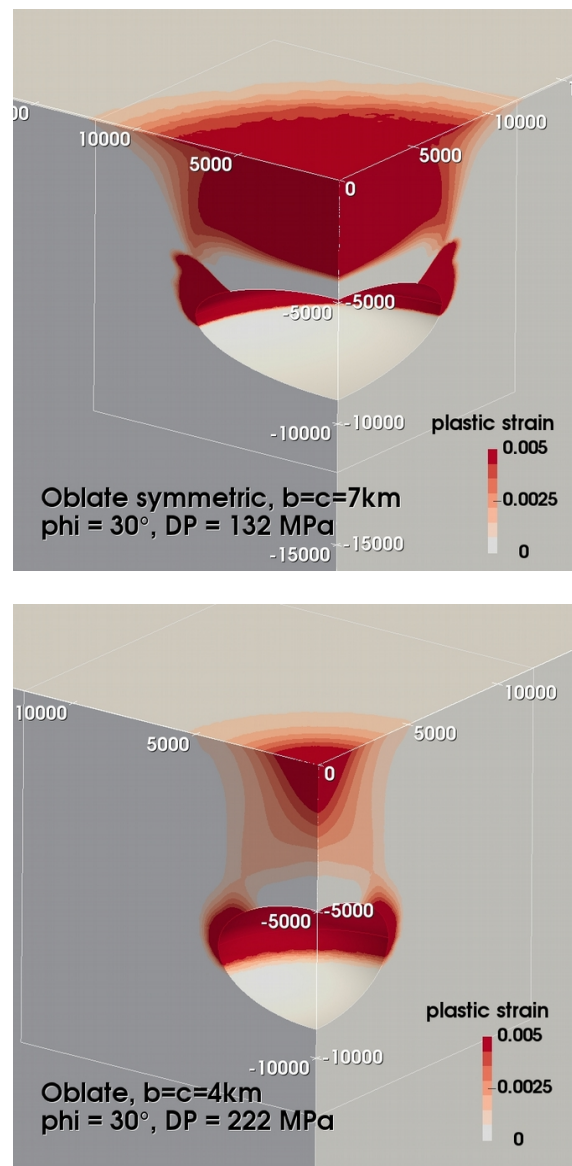
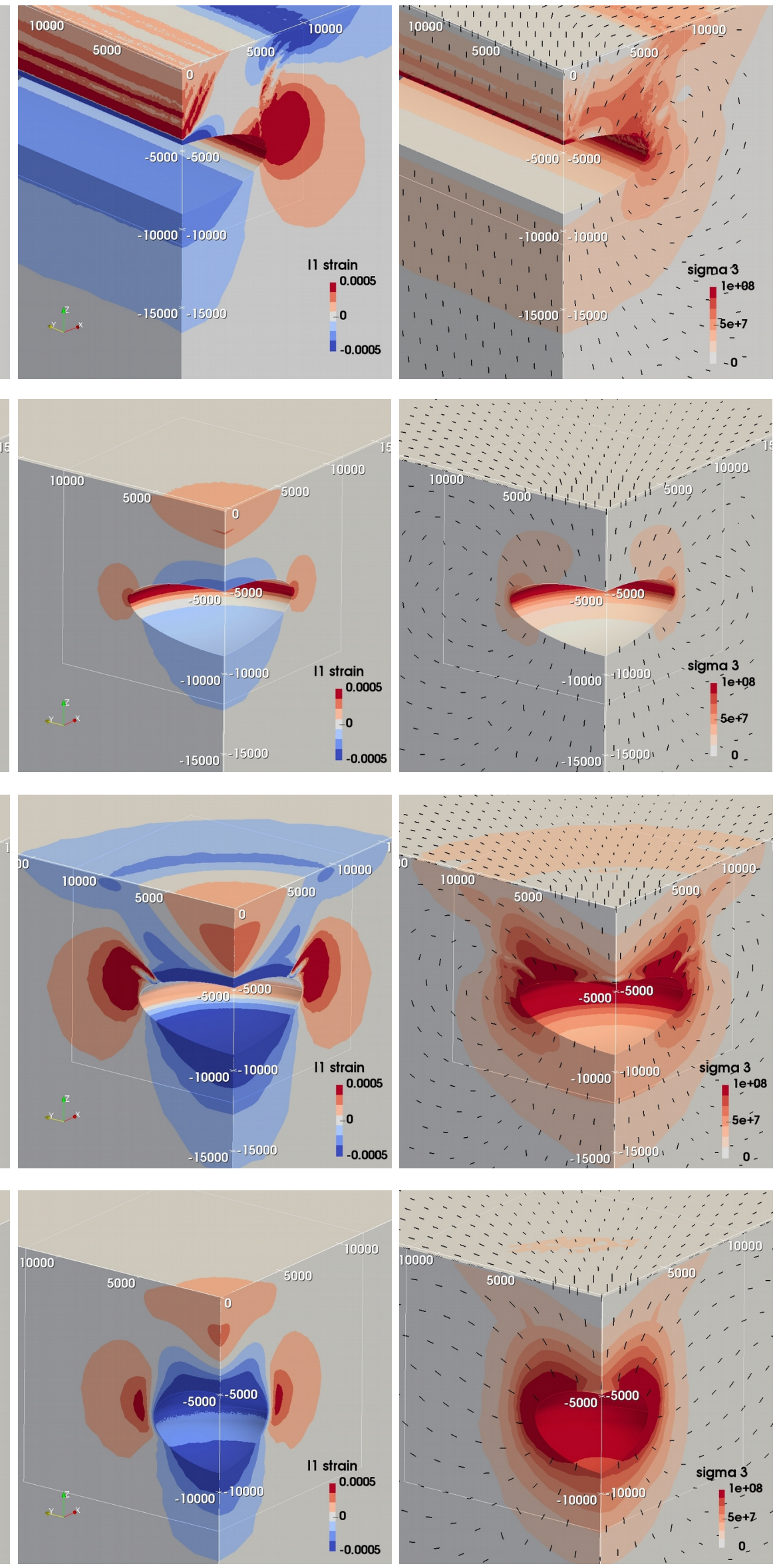
7a)

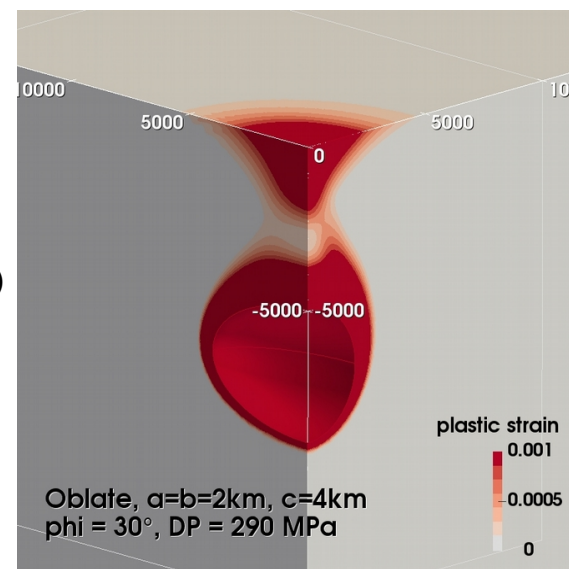

7b)

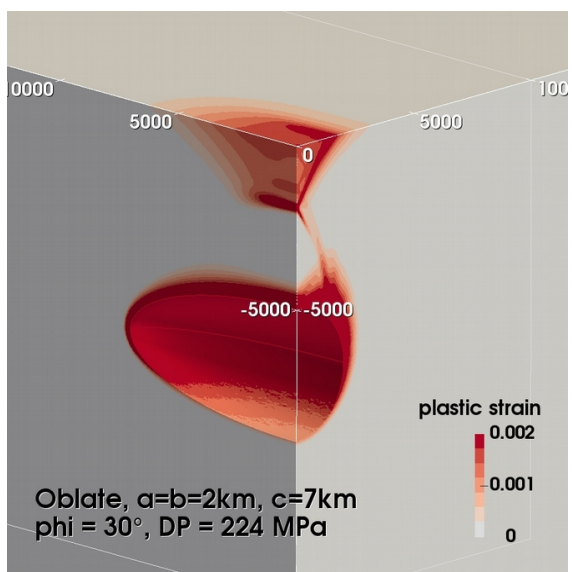

7c)

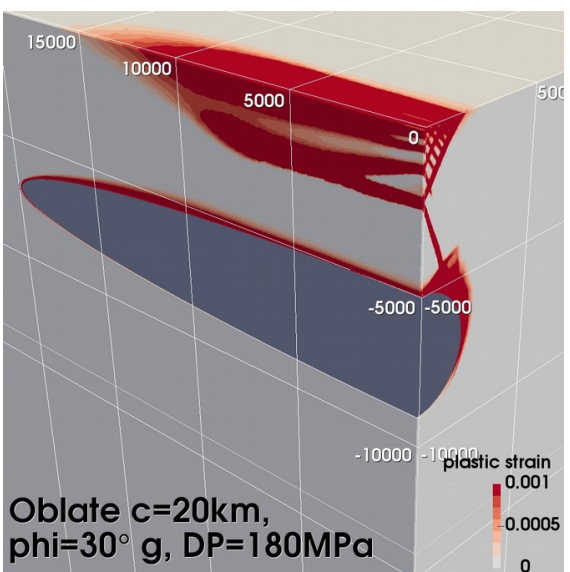

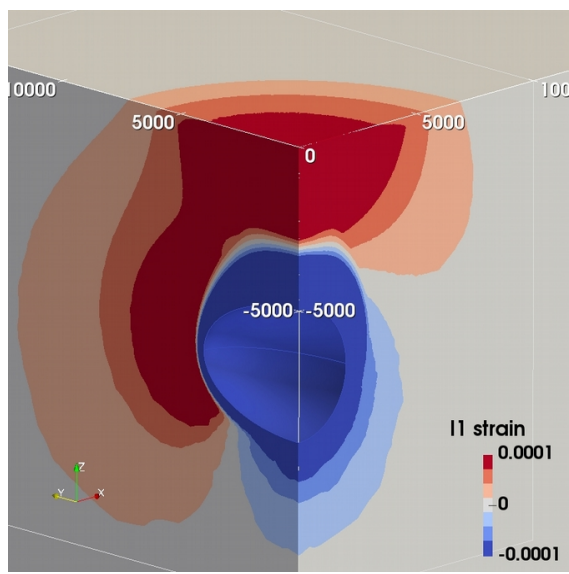
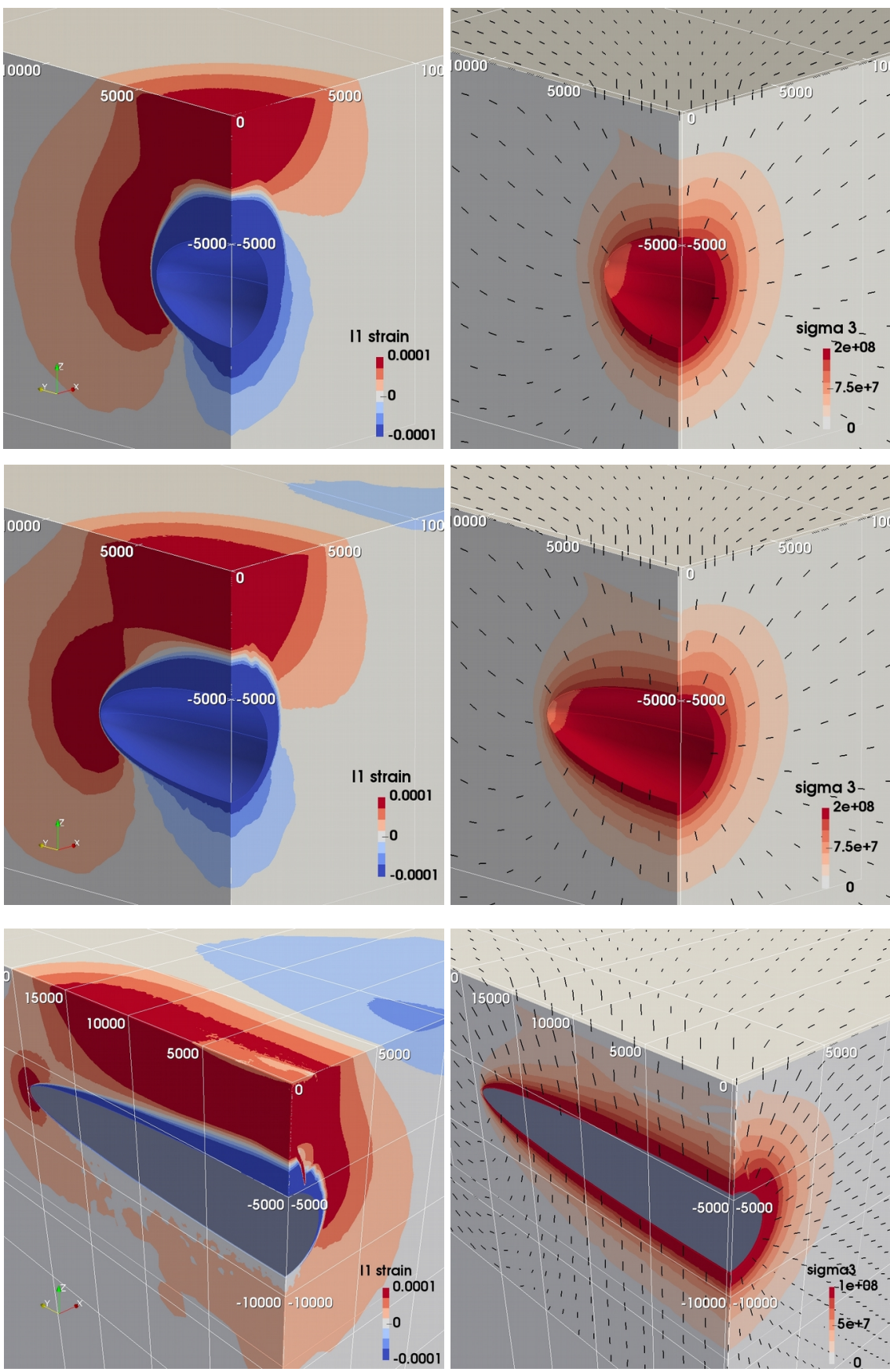

This article is protected by copyright. All rights reserved. 
8a)
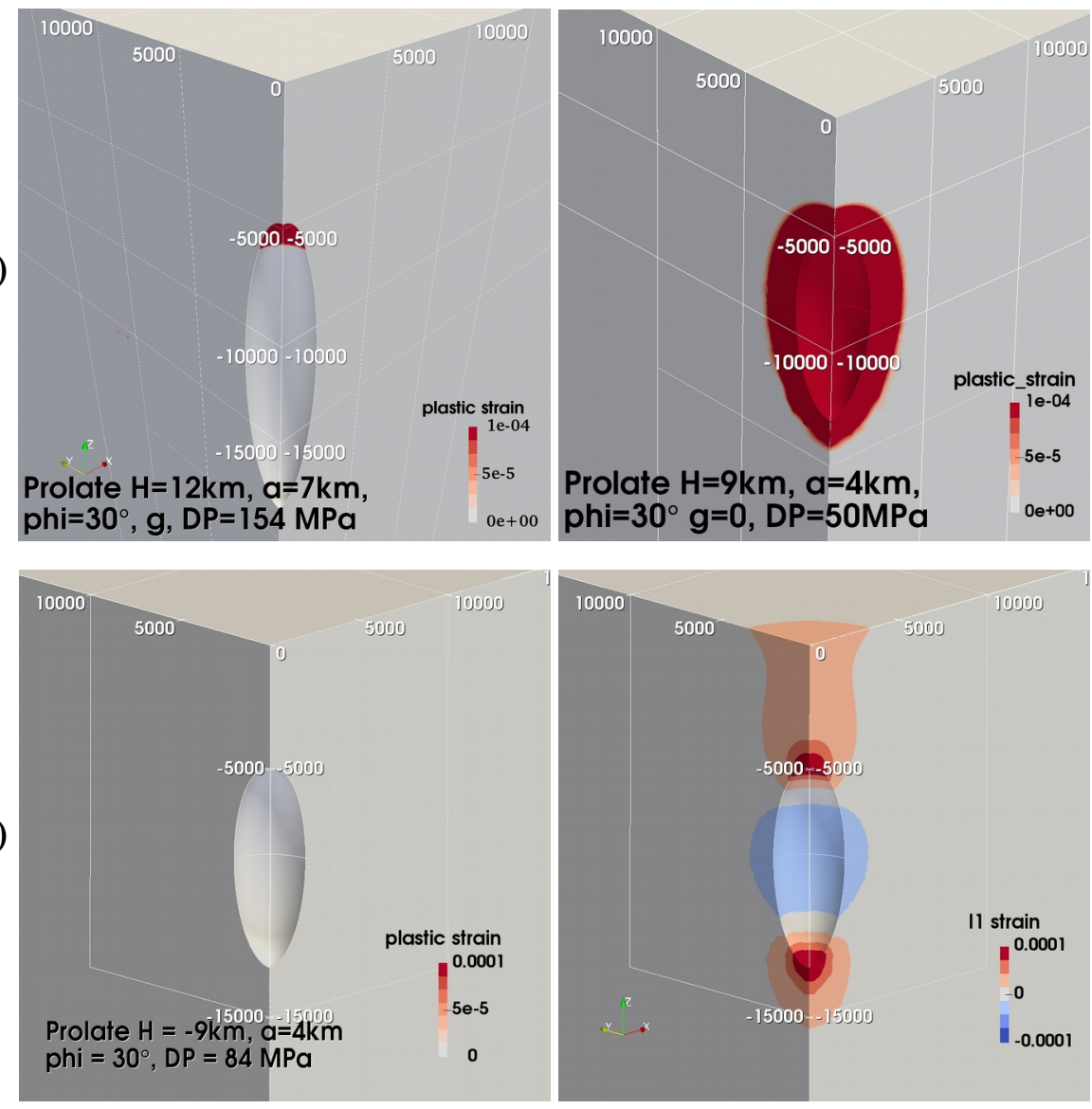

Prolate $H=-9 \mathrm{~km}, \mathrm{a}=4 \mathrm{~km}$ phi $=30^{\circ}, \mathrm{DP}=84 \mathrm{MPa}$

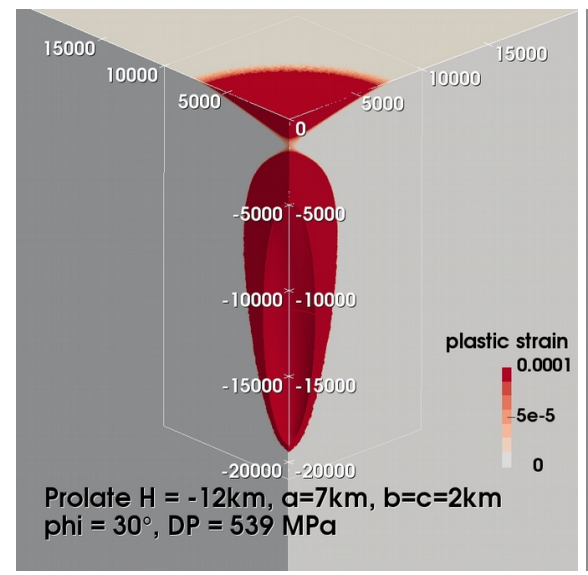

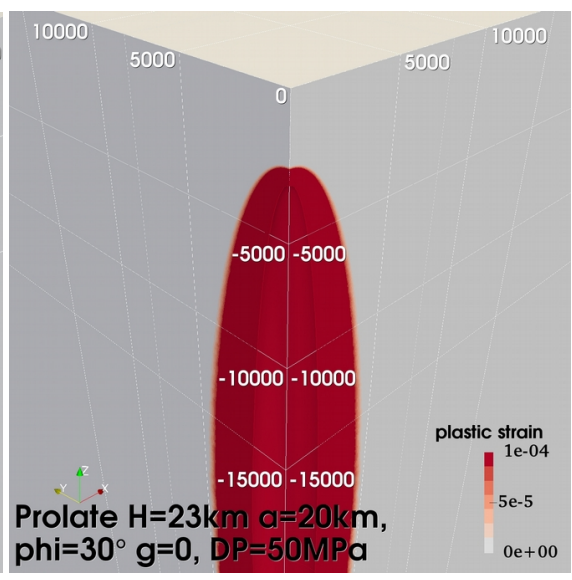
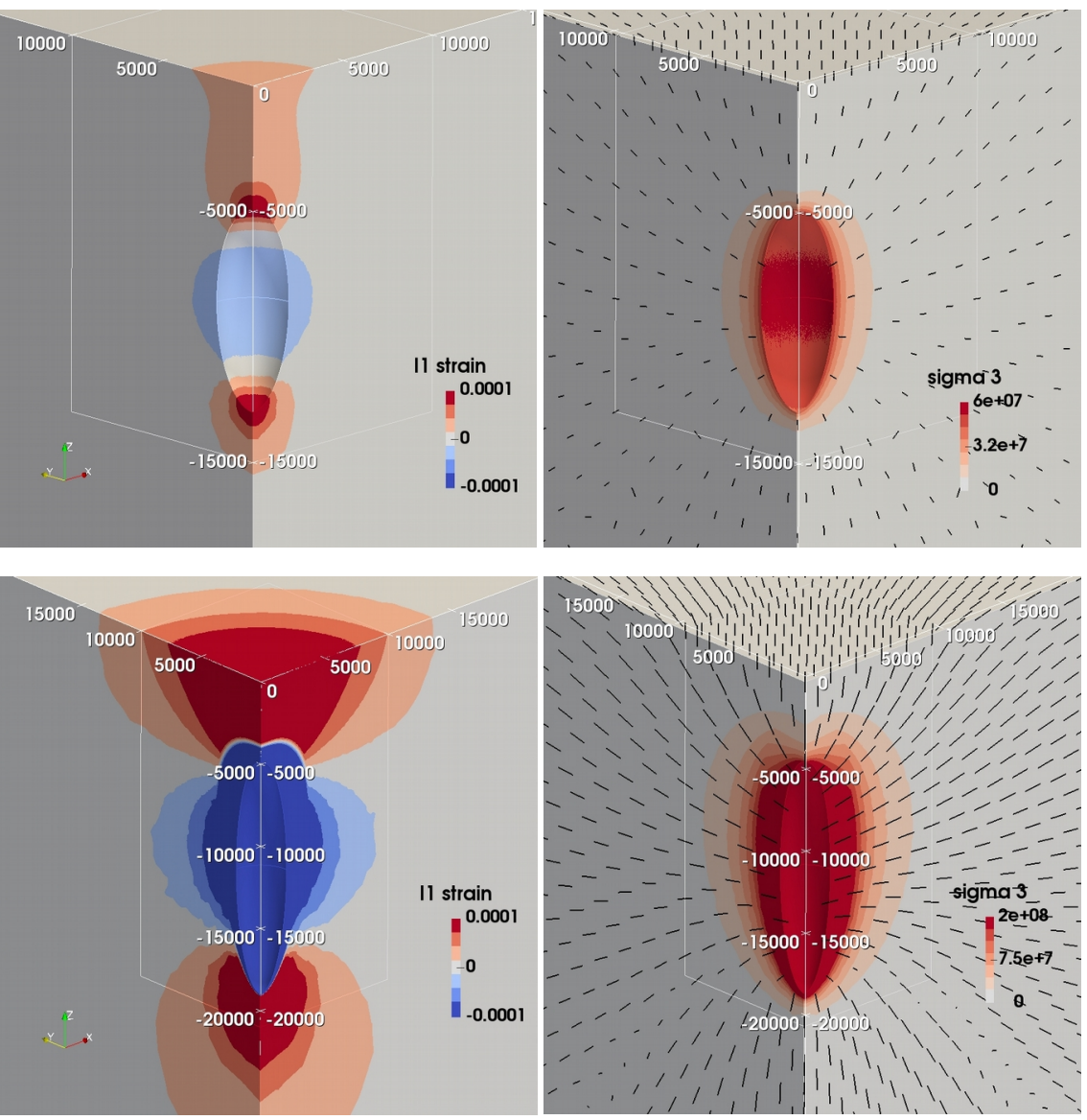

This article is protected by copyright. All rights reserved. 

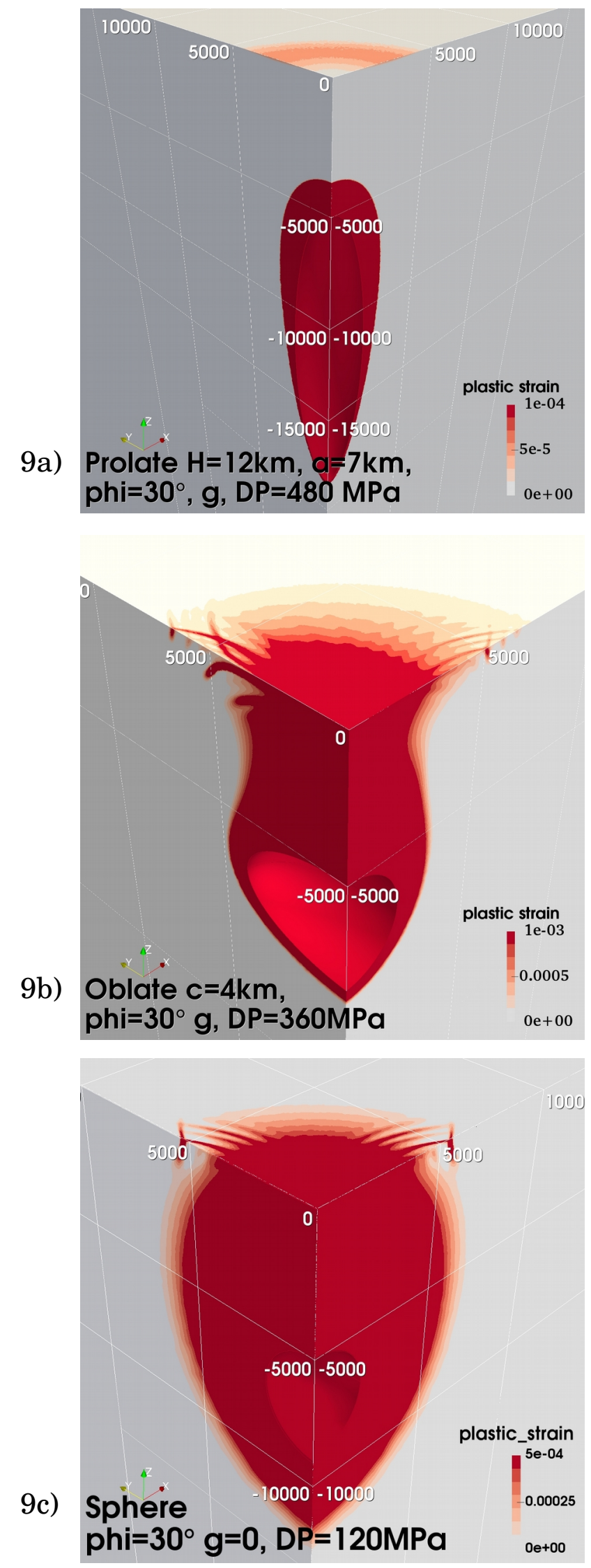

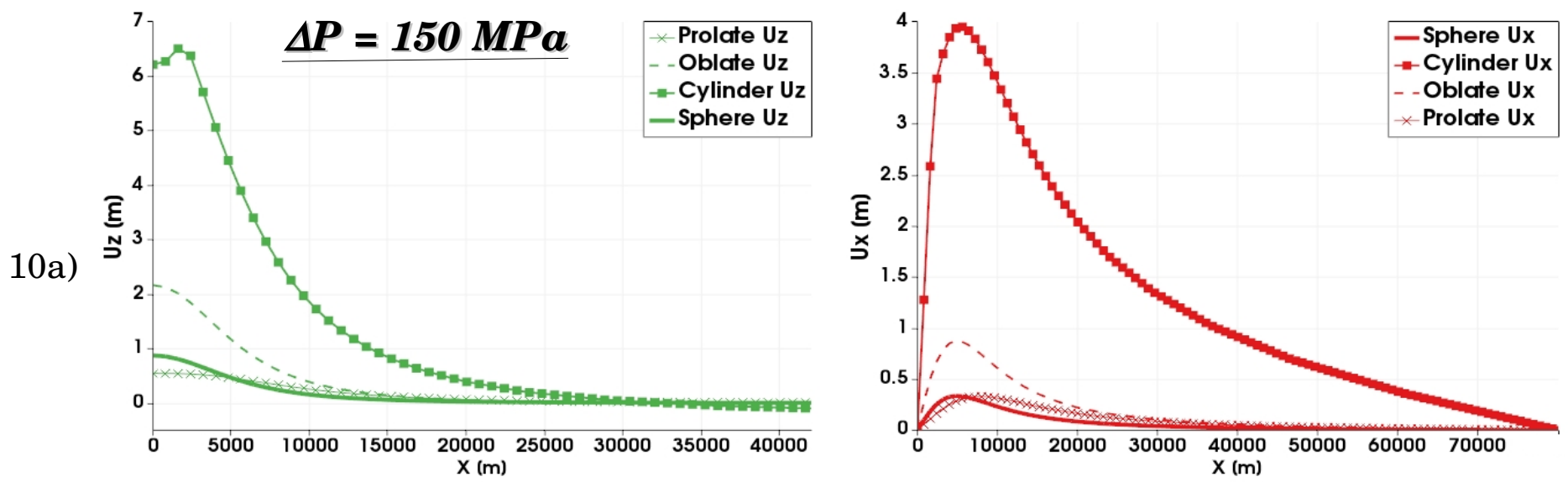

10b)
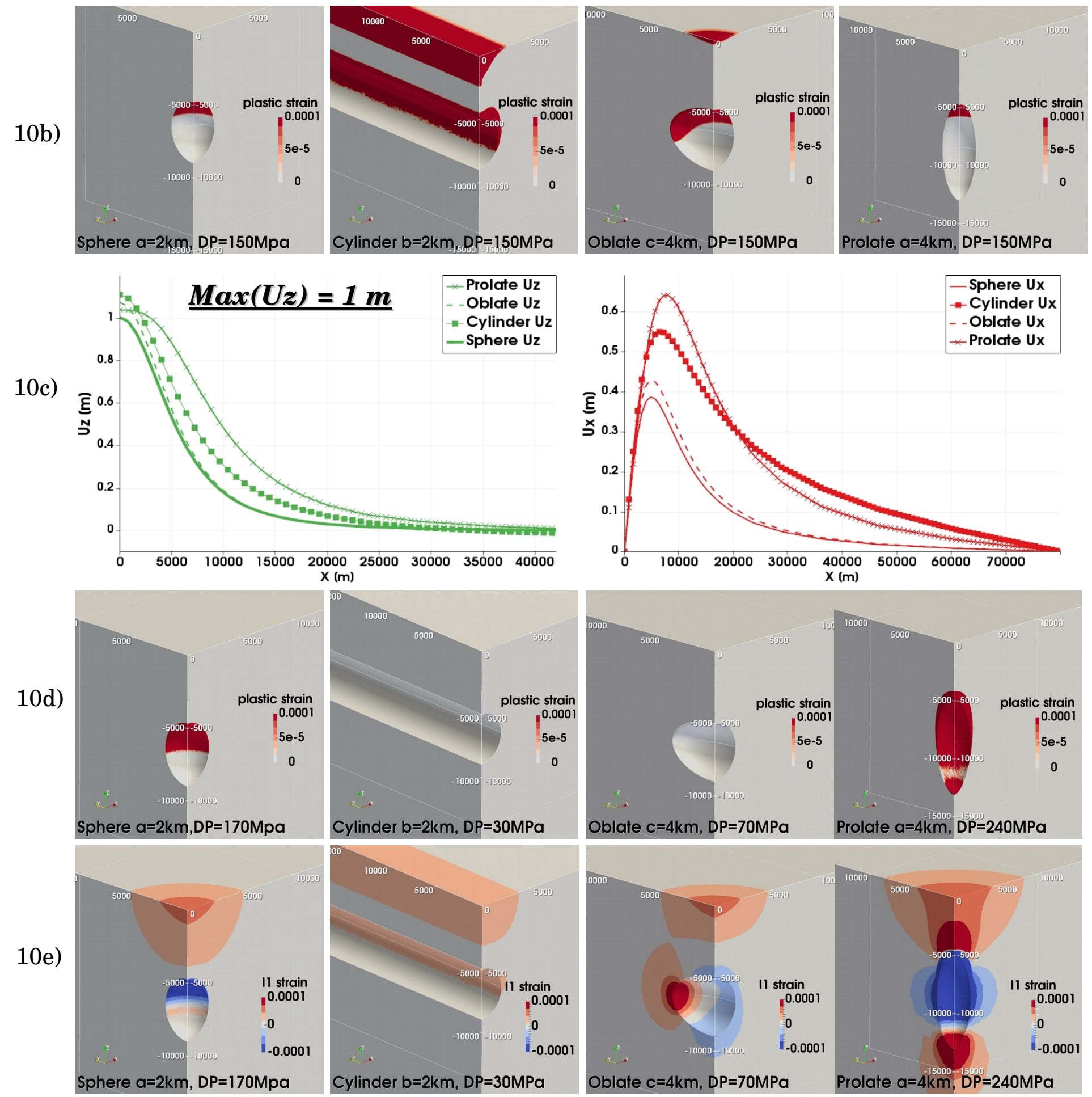

This article is protected by copyright. All rights reserved. 